\title{
X-ray spectral properties of Seyfert galaxies and the unification scheme
}

\author{
V. Singh ${ }^{1,2}$, P. Shastri ${ }^{1}$, and G. Risaliti ${ }^{3,4}$ \\ 1 Indian Institute of Astrophysics, Bangalore 560034, India \\ e-mail: veeresh@iiap.res.in \\ 2 Department of Physics, University of Calicut, Calicut 673635, India \\ 3 INAF-Osservatorio di Arcetri, Largo E. Fermi 5, 50125 Firenze, Italy \\ 4 Harvard-Smithsonian Center for Astrophysics, 60 Garden St. Cambridge, MA 02138, USA
}

Received 22 December 2010 / Accepted 1 April 2011

\begin{abstract}
Context. The unification scheme of Seyfert galaxies predicts that the observed differences between types 1 and 2 Seyfert galaxies are solely due to the differing orientations of the toroidal-shaped obscuring material around AGN. The observed X-ray spectra of Seyfert type 2 s compared to type 1 s are expected to be affected by higher absorbing column density owing to the edge-on view of the obscuring torus.

Aims. We study the $0.5-10 \mathrm{keV}$ X-ray spectral properties of Seyfert type $1 \mathrm{~s}$ and type $2 \mathrm{~s}$ with the aim of testing the predictions of Seyfert unification scheme in the X-ray regime.

Methods. We use an optically selected Seyfert sample in which type $1 \mathrm{~s}$ and type $2 \mathrm{~s}$ have matched distributions in parameters that are independent of the orientation of AGN and the host galaxy.

Results. The 0.5-10 keV XMM-Newton pn X-ray spectra of Seyfert galaxies are in general best fitted with a model that consists of (i) an absorbed powerlaw; (ii) a narrow Gaussian fitted to the $\mathrm{Fe} \mathrm{K} \alpha$ emission line; and (iii) an often seen soft-excess component characterized by either a thermal plasma model with temperature $k T \sim 0.1-1.0 \mathrm{keV}$ or a steep powerlaw or both. The $2.0-10 \mathrm{keV}$ hard X-ray continuum emission in several Seyfert type $2 \mathrm{~s}$ is dominated by reflection and suggests the Compton-thick obscuration. Results of the statistical comparison between the distributions of (i) the observed X-ray luminosities in the soft $(0.5-2.0 \mathrm{keV})$ and hard (2.0-10.0 keV) bands; (ii) the X-ray absorbing column densities; (iii) the equivalent widths of $\mathrm{Fe} \mathrm{K} \alpha$ line; and (iv) the flux ratios of hard X-ray to [OIII] $\lambda 5007 \AA$, for the two Seyfert subtypes are consistent with the obscuration and orientation-based unification scheme.
\end{abstract}

Key words. galaxies: Seyfert - X-rays: galaxies - galaxies: active

\section{Introduction}

Seyfert galaxies are mainly classified into two classes, based on the presence (type 1) or absence (type 2) of broad emission lines in their optical spectra (Antonucci 1993). The unification scheme of Seyfert galaxies hypothesizes that Seyfert types 1s and $2 \mathrm{~s}$ constitute the same parent population and appear different solely due to the differing orientations of the AGN axis with respect to the line-of-sight. The scheme requires the presence of toroidal-shaped dusty molecular obscuring material around the AGN. In the type $2 \mathrm{~s}$, this torus is inclined edge-on and blocks the direct view to the nuclear region, while in type 1s the observer's line-of-sight is away from the plane of the torus, and the central region is visible (Antonucci \& Miller 1985; Antonucci 1993; Urry et al. 1995).

Several investigations in the literature have yielded results that are consistent with the predictions of this scheme, such as, the presence of broad emission lines in the polarized optical and infrared spectra of many Seyfert 2s (Moran et al. 2000), the biconical structure of the narrow line region (Mulchaey et al. 1996), the similarity of CO masses of the two Seyfert subtypes (Maiolino et al. 1997), and similar nuclear radio properties of both the subtypes (Lal et al. 2011). Inconsistencies in the predictions of the scheme remain, however, such as, the absence of hidden Seyfert 1 nuclei in several Seyfert 2s (Tran 2001, 2003), higher tendency for Seyfert 1s to be hosted in galaxies of earlier Hubble type (Malkan et al. 1998), the lack of X-ray absorption in some Seyfert 2s (Panessa \& Bassani 2002), and higher propensity of nuclear starbursts in Seyfert 2s (Buchanan et al. 2006). Moreover, it has been evident that the sample selection plays a crucial role in testing the predictions of Seyfert unification scheme (Antonucci 2002). Ho \& Ulvestad (2001) argued that the optical and UV selected samples are likely to have inherent biases against the obscured sources. Infrared selected samples can be biased towards unusually dusty sources as well as towards those with a higher level of nuclear star formation (Ho \& Ulvestad 2001; Buchanan et al. 2006). X-ray selected samples have also been used to examine the validity of Seyfert unification (Awaki et al. 1991; Smith \& Done 1996; Turner et al. 1997a,b, 1998; Bassani et al. 1999). However, the Seyfert samples selected from flux-limited surveys are likely to have obscured type 2 Seyferts that are intrinsically more luminous than the selected type 1 counterparts. Maiolino et al. (1998) and Risaliti et al. (1999) have shown the increased number of heavily obscured type 2 sources using a sample based on [OIII] $\lambda 5007 \AA$ line luminosity, which suggests the generally inherent bias against less luminous and heavily obscured sources in X-ray selected samples. Hard X-ray selected samples are supposed to be less biased but cannot be claimed to be free of biases against heavily obscured Compton-thick and 
low luminosity AGNs (Heckman et al. 2005; Wang et al. 2009). Recent Seyfert samples based on INTEGRAL and Swift/BAT surveys primarily contain relatively large number of high luminosity and less absorbed Seyferts (Tueller et al. 2008; Treister et al. 2009; Beckmann et al. 2009), possibly due to the less effective area that limits the sensitivity to only bright sources $\left(\sim 10^{-11} \mathrm{erg} \mathrm{s}^{-1} \mathrm{~cm}^{-2}\right)$. The quest to test the validity and limitations of the Seyfert unification with more improved and welldefined samples continues. Recent studies by Cappi et al. (2006); Dadina (2008), and Beckmann et al. (2009) have used lessbiased optically and X-ray-selected samples and report results that are broadly consistent with unification; nonetheless, issues related to sample selection still remain. With the above sampleselection arguments in mind, we opted to use a sample in which Seyfert types $1 \mathrm{~s}$ and $2 \mathrm{~s}$ have matched distributions of parameters that are independent to the orientation of the obscuring torus and AGN axis. Such sample selection mitigates the biases generally inherent in flux-limited surveys and also ensures that the two Seyfert subtypes are intrinsically similar within the framework of the Seyfert unification scheme (Schmitt et al. 2003; Lal et al. 2011).

In the standard picture of the unification scheme, AGN powered by accretion onto a supermassive black hole and the surrounding broad line region clouds are embedded in an obscuring torus (Antonucci \& Miller 1985; Antonucci 1993; Urry et al. 1995). The X-ray emission originating from the AGN carries the imprints of obscuring and reprocessing material, therefore Seyfert X-ray spectra are expected to differ depending upon whether the AGN is viewed directly (i.e., type 1s) or through the obscuring torus (i.e., type $2 \mathrm{~s}$ ). In Seyfert type $2 \mathrm{~s}$, the intrinsic X-ray emission is attenuated by the obscuring torus and the observed flux, as well as the X-ray spectral shape, depend on the optical depth of the obscuring torus (Awaki et al. 1991; Smith \& Done 1996; Turner et al. 1997a; Bassani et al. 1999; Cappi et al. 2006). Since the photoelectric absorption crosssection decreases with the increase in photon energy, an increasing fraction of primary high-energy photons are expected to transmit through the torus. Therefore, Seyfert $1 \mathrm{~s}$ and $2 \mathrm{~s}$ are expected to show systematically less difference for the observed hard X-ray luminosities and more difference for the soft $\mathrm{X}$-ray $(E<2.0 \mathrm{keV})$ luminosities. Since in type $2 \mathrm{~s}$, the AGN is viewed through the obscuring torus, type $2 \mathrm{~s}$ are expected to show systematically higher absorbing column densities than type $1 \mathrm{~s}$. The absorbing column density can be measured via photoelectric absorption component in $0.5-10 \mathrm{keV}$ spectral fits as long as obscuration is Compton-thin $\left(N_{\mathrm{H}}<10^{24} \mathrm{~cm}^{-2}\right)$. If obscuration is Compton-thick $\left(N_{\mathrm{H}}>10^{24} \mathrm{~cm}^{-2}\right)$, type $2 \mathrm{~s}$ become faint even at higher X-ray energies since high energy photons are Compton-down scattered to soft photons, which eventually get absorbed or scattered out of the line-of-sight (Matt et al. 2000a). The equivalent width ( $E W$ ) of $\mathrm{Fe} \mathrm{K} \alpha$ line also increases with the absorbing column density because it is measured against the depressed continuum, and it increases to $\sim 1 \mathrm{keV}$ for Compton-thick sources (Ghisellini et al. 1994; Levenson et al. 2006). The flux ratio of hard X-ray (2.0-10 keV) to [OIII] $\lambda 5007 \AA$ line emission can also be used as a diagnostic parameter for the amount of obscuration, and type $2 \mathrm{~s}$ are expected to show lower flux ratios of hard X-ray to [OIII] than type 1s (Bassani et al. 1999; Cappi et al. 2006).

In this paper we test these predictions of the unification scheme by comparing properties, such as the observed soft and hard X-ray luminosities, the X-ray absorbing column densities, the $E W \mathrm{~s}$ of $\mathrm{Fe} \mathrm{K} \alpha$ line, and the flux ratios of $\mathrm{X}$-ray to [OIII] $\lambda 5007 \AA$ for the Seyfert types 1s and 2s. In other words, using 0.5-10 keV XMM-Newton data, we test whether $\mathrm{X}$-ray properties conform with the optical classification of Seyfert types $1 \mathrm{~s}$ and $2 \mathrm{~s}$ in the framework of the orientation and obscuration-based unification scheme. The important aspect of our study is the sample selection in which types $1 \mathrm{~s}$ and $2 \mathrm{~s}$ are matched in orientation-independent parameters.

A description of the sample, observations, and data reduction is given in Sects. 2 and 3, while Sect. 4 describes the spectral modeling of X-ray spectra. The statistical comparisons of X-ray spectral properties of the two Seyfert subtypes are discussed in Sect. 5. Notes on individual Seyfert galaxies are given in Appendix A. In this paper, we assume $H_{0}=71 \mathrm{~km}^{-1} \mathrm{Mpc}^{-1}$, $\Omega_{\mathrm{m}}=0.27$, and $\Omega_{\mathrm{vac}}=0.73$.

\section{The sample}

We used the sample of Lal et al. (2011), which consists of 20 (10 type 1 and 10 type 2 ) optically selected Seyfert galaxies. This sample was formulated to study the nuclear radio properties in the framework of Seyfert unification scheme, and in this paper we attempt to test the predictions of Seyfert unification scheme in the X-ray regime using the same sample. Although the sample was constructed to investigate the parsec-scale radio emission and is constrained by VLBI observing feasibility criteria, it is adequately qualified to test the predictions of the unification scheme in the X-ray regime, since it is based on the orientationindependent parameters. In this sample Seyfert galaxies are defined as radio quiet $\left(\frac{F_{5} \mathrm{GHz}}{F_{B \text {-band }}}<10\right.$; Kellermann et al. 1989), low optical luminosity $\operatorname{AGN}\left(M_{B}>-23\right.$; Schmidt \& Green 1983), hosted in spiral or lenticular galaxies (Weedman 1977). All the intermediate subclasses of Seyferts (i.e., 1.0, 1.2, 1.5, 1.8, 1.9), which show any broad permitted emission line component in their optical spectra, are grouped as type $1 \mathrm{~s}$, while those that show only narrow permitted emission lines are considered as type 2 Seyferts. The sample was selected such that the two Seyfert subtypes have matched distributions in the orientationindependent parameters of AGN and host galaxy, and the sources that deviated in matching the type 1 and type 2 distributions of the orientation-independent parameters were left out. In other words, the sources should lie within the same range of values for a given parameter to enter into the sample. Also, it was ensured that type $1 \mathrm{~s}$ do not outnumber the type $2 \mathrm{~s}$ and vice-versa in a given bin of a parameter distribution. Indeed, there is a possibility of increasing the sample size following the same sample selection criteria. However, we would like to emphasize that the sample selection criteria are more important and not the sample size for rigorously testing the predictions of the unification scheme. A larger but heterogeneous and biased sample is likely to result incorrect conclusions.

Cosmological redshift, [OIII] $\lambda 5007 \AA$ line luminosity, Hubble stage of the host galaxy, absolute stellar magnitude of the host galaxy, and absolute magnitude of the bulge are considered as the orientation-independent properties for the sample selection. These properties do not depend on the orientation of the obscuring torus, AGN axis, and the host galaxy. And, indeed all these properties are intimately linked to the evolution of AGN, as well as to the host galaxy. We refer the reader to Lal et al. (2011) for the detailed description of the sample selection and of the matching of the distributions of the orientation-independent parameters for the two Seyfert subtypes. The matching of types 1s and $2 \mathrm{~s}$ in orientation-independent isotropic parameters ensures that we are not comparing entirely intrinsically different sources 
Table 1. XMM-Newton EPIC pn observations log.

\begin{tabular}{lccccccc}
\hline \hline $\begin{array}{l}\text { Source } \\
\text { name }\end{array}$ & $\begin{array}{c}\text { Obs. } \\
\text { mode/filter }\end{array}$ & $\begin{array}{c}\text { Obs. } \\
\text { date }\end{array}$ & $\begin{array}{c}\text { Obs. } \\
\text { time }(\mathrm{ks})\end{array}$ & $\begin{array}{c}\text { Net expo. } \\
\text { time }(\mathrm{ks})\end{array}$ & ct/s & Obs. ID & $\begin{array}{c}R_{\mathrm{S}} \\
(\mathrm{arcsec})\end{array}$ \\
\hline MCG+8-11-11 & SW/Medium & $2004-04-10$ & 38.45 & 25.24 & 14.93 & 0201930201 & 35.0 \\
MRK 1218 & SW/Thin & $2005-04-09$ & 13.87 & 6.55 & 0.53 & 0302260201 & 20.0 \\
NGC 2639 & FW/Thin & $2005-04-03$ & 26.12 & 4.23 & 0.24 & 0301651101 & 16.0 \\
NGC 4151 & SW/Medium & $2006-11-29$ & 52.81 & 24.60 & 9.57 & 0402660201 & 40.0 \\
MRK 766 & SW/Medium & $2005-05-24$ & 95.51 & 60.12 & 4.92 & 0304030101 & 40.0 \\
MRK 231 & FW/Medium & $2001-06-07$ & 22.34 & 17.30 & 0.10 & 0081340201 & 30.0 \\
ARK 564 & SW/Medium & $2005-01-06$ & 101.77 & 69.07 & 39.67 & 0206400101 & 35.0 \\
NGC 7469 & SW/Medium & $2004-12-03$ & 79.11 & 54.95 & 21.59 & 0207090201 & 37.5 \\
MRK 926 & SW/Thin & $2000-12-01$ & 11.76 & 7.19 & 18.33 & 0109130701 & 37.5 \\
MRK 530 & SW/Thick & $2006-06-14$ & 16.82 & 11.42 & 12.93 & 0305600601 & 20.0 \\
MRK 348 & FW/Medium & $2002-07-18$ & 49.50 & 26.54 & 1.96 & 0067540201 & 22.5 \\
MRK 1 & FW/Thin & $2004-01-09$ & 11.91 & 8.66 & 0.07 & 0200430301 & 17.5 \\
NGC 2273 & FW/Medium & $2003-09-05$ & 13.02 & 5.21 & 0.08 & 0140951001 & 20.0 \\
MRK 78 & FW/Thin & $2006-03-11$ & 16.12 & 4.83 & 0.09 & 0307001501 & 22.5 \\
NGC 7212 & FW/Thin & $2004-05-20$ & 14.22 & 10.50 & 0.10 & 0200430201 & 20.0 \\
MRK 533 & LW/Thick & $2004-06-02$ & 10.42 & 7.51 & 0.12 & 0200660101 & 17.5 \\
NGC 7682 & FW/Thin & $2005-05-27$ & 20.21 & 14.27 & 0.04 & 0301150501 & 16.0 \\
\hline
\end{tabular}

Notes. SW: small window; FW: full window; LW: large window; $R_{\mathrm{S}}$ : radius of the circular region around the source used for spectral extraction.

selected from different parts of the evolution function (luminosity, bulge mass, Hubble type, redshift).

\section{X-ray observations and data reduction}

We modeled the X-ray spectra of our sample sources using XMM-Newton EPIC-pn observations obtained from the $X M M-N e w t o n$ Science Archive. High throughput (especially, at energies $E \geq 2.0 \mathrm{keV}$ ) of XMM-Newton allows us to search for spectral components with absorbing column density up to $N_{\mathrm{H}} \sim 10^{24} \mathrm{~cm}^{-2}$, and its high spatial resolution minimizes any strong contamination from off-nuclear X-ray emission. For our $\mathrm{X}$-ray spectral study, we used only EPIC pn data and did not include EPIC MOS data, since it is more susceptible to pileup for bright X-ray sources and a few sources; e.g., MRK 530 do not have MOS data. Also, EPIC pn data is good enough for our purpose of obtaining the average X-ray spectral parameters of our sample sources (e.g., photon index, equivalent hydrogen column density, $E W$ of $\mathrm{Fe} \mathrm{K} \alpha$ emission line). When a source had more than one observations in the archive, we chose the latest one with long enough exposure time. The observation log is given in Table 1, and it gives the details of observing modes of pn camera, the optical light blocking filters, observation dates, observation and net exposure times, count rates, observation IDs, and radius (in arcsec) of the circular region around the source position used to extract the X-ray spectra. The raw observation data files (ODFs) were reduced and analyzed using the standard Science Analysis System (SAS) software package (version 7.1) with the latest calibration files. The time intervals of high-energy background particle flares were removed by applying a fixed threshold on single events, using $E \geq 10.0 \mathrm{keV}$, and $\delta t=50 \mathrm{~s}$ light curves. The background is extracted from a circular region in the same chip. To apply $\chi^{2}$ statistics, the spectra were binned with minimum 20 counts per bin.

We present the X-ray spectra of 17 (10 type 1 s and 7 type 2 s) out of 20 Seyfert galaxies in the sample using XMM-Newton pn archival data. For six Seyfert galaxies, i.e., MRK 1218, NGC 2639, MRK 530, MRK 78, MRK 533, and NGC 7682, the XMM-Newton X-ray spectra are presented for the first time, and for two Seyferts, i.e., NGC 4151 and NGC 7469, the XMM-Newton archival data chosen by us have not been published. The XMM-Newton spectra of the rest of the nine sources have been individually published; however, to have a uniform reduction and analysis, we re-did the X-ray spectral fitting of these sources using data reduced with the most updated calibration files. Also, we did not rule out the possibility of obtaining the different or better fits for these nine sources, and therefore we preferred to re-do the spectral analysis for these sources. For three (type 2) Seyfert galaxies of our sample, i.e., NGC 5135, MRK 477, and NGC 5929, there are no XMMNewton data, and we used the Chandra and ASCA spectral parameters for these sources from the literature, while performing the statistical comparison of the X-ray properties of the two subtypes of Seyferts.

\section{X-ray spectral analysis}

We attempted to obtain the physically motivated best fits for the $0.5-10.0 \mathrm{keV}$ XMM-Newton pn X-ray spectra of our sample Seyfert galaxies. Since the primary aim of spectral analysis is to identify the underlying continuum components, we first tried to fit each spectrum with the simplest model, which consists of a powerlaw plus absorption fixed at the Galactic value, and an intrinsic absorption as a free parameter. However, in most cases this simple parametrization is insufficient to model the whole spectrum and residuals often show a soft excess and an emission line feature at $6.4 \mathrm{keV}$ on top of the (absorbed or unabsorbed) powerlaw. In most cases, we found the best-fit model consists of: a powerlaw with a photoelectric cutoff due to cold absorption from the Galactic column density and neutral gas at the redshift of the source; a narrow Gaussian line to reproduce the iron $\mathrm{K} \alpha$ fluorescent emission line at $6.4 \mathrm{keV}$ in the rest frame with energy, width, and intensity as free parameters; a soft-excess component characterized by either a steep powerlaw and/or a thermal plasma model with temperature $k T$. In some cases, a reflection component characterized by reflection from an isotropically illuminated cold slab (model PEXRAV Magdziarz \& Zdziarski 1995; in XSPEC Arnaud 1996) is required for the best fit. A partial covering of the primary powerlaw component is also needed for the best fit in some sources. The partial covering model is equivalent to a combination of an absorbed powerlaw plus a scattered powerlaw, assuming that the powerlaw 
Table 2. The best-fit spectral parameters.

\begin{tabular}{|c|c|c|c|c|c|c|c|c|c|c|}
\hline \multirow[t]{2}{*}{ Source } & \multirow[t]{2}{*}{ Model } & \multirow[b]{2}{*}{$\begin{array}{c}N_{\mathrm{H}} \\
\left(10^{20} \mathrm{~cm}^{-2}\right)\end{array}$} & \multicolumn{2}{|c|}{ Soft X-ray continuum } & \multicolumn{2}{|c|}{ "Hard X-ray continuum } & \multicolumn{3}{|c|}{ Emission lines } & \multirow[t]{2}{*}{$\chi^{2} /$ d.o.f } \\
\hline & & & $\Gamma_{\mathrm{SX}}$ & $\begin{array}{c}k T \\
(\mathrm{keV})\end{array}$ & $\begin{array}{c}N_{\mathrm{H}, \text { hard }} \\
\left(10^{22} \mathrm{~cm}^{-2}\right)\end{array}$ & $\Gamma_{\mathrm{HX}}$ & $\begin{array}{l}E_{\text {c,rest }} \\
(\mathrm{keV})\end{array}$ & $I_{\text {line }}$ & $\begin{array}{l}E W \\
(\mathrm{eV})\end{array}$ & \\
\hline$\overline{\mathrm{MCG}+8-11-11}$ & pha(PL+R+L) & $18.30_{-0.43}^{+0.40}$ & & & & $1.84_{-0.02}^{+0.02}$ & $6.44_{-0.01}^{+0.01}$ & 4.95 & $97.9_{-18.5}^{+10.4}$ & 0.99 \\
\hline MRK 1218 & pha(abs*PL+L) & $15.20_{-13.00}^{+4.30}$ & & & & $1.48_{-0.17}^{+0.11}$ & $6.32_{-0.49}^{+0.21}$ & 0.19 & $63.9_{-52.4}^{+46.5}$ & 0.99 \\
\hline NGC 2639 & pha $(\mathrm{T}+\mathrm{PL})$ & $16.20_{-15.60}^{+17.60}$ & & $0.63_{-0.09}^{+0.12}$ & & $2.21_{-1.02}^{+1.83}$ & & & & 0.82 \\
\hline NGC 4151 & pha $(\mathrm{T}+\mathrm{PL}+$ pha*abs*pcf*PL+R+L+L) & $6.33_{-2.60}^{+3.30}$ & $3.14_{-0.06}^{+0.25}$ & $0.13_{-0.01}^{+0.01}$ & $0.12_{-0.03}^{+0.08}$ & $1.75_{-0.03}^{+0.06}$ & $\begin{array}{l}6.39_{-0.01}^{+0.01} \\
0.88_{-0.01}^{+0.01}\end{array}$ & $\begin{array}{c}14.43 \\
1.12\end{array}$ & $\begin{array}{l}108.4_{-6.7}^{+6.5} \\
60.1_{-4.4}^{+29.0}\end{array}$ & 1.12 \\
\hline MRK 766 & pha $(\mathrm{T}+\mathrm{abs} * \mathrm{pcf} * \mathrm{PL}+\mathrm{R}+\mathrm{L}+\mathrm{L}+\mathrm{L})$ & $1.35^{f}$ & & $0.20_{-0.01}^{+0.01}$ & & $2.17_{-0.08}^{+0.10}$ & $\begin{array}{r}6.44_{-0.03}^{+0.01} \\
6.67_{-0.07}^{+0.06} \\
0.48_{-0.01}^{+0.01}\end{array}$ & $\begin{array}{l}0.50 \\
0.22 \\
5.38\end{array}$ & $\begin{array}{c}-4.4 \\
47.9_{-13.5}^{+13.9} \\
19.3_{-12.2}^{+12.6} \\
137.3_{-375}^{+45.6}\end{array}$ & 1.09 \\
\hline MRK 231 & pha $(\mathrm{T}+\mathrm{T}+$ pcf*pha*PL+L $+\mathrm{L})$ & $1.25^{f}$ & & $\begin{array}{l}0.33_{-0.03}^{+0.04} \\
0.99_{-0.15}^{+0.14}\end{array}$ & $0.46_{-0.16}^{+0.14}$ & $1.57_{-0.30}^{+0.14}$ & $\begin{array}{l}6.28_{-0.13}^{+0.13} \\
6.66_{-0.20}^{+0.07}\end{array}$ & $\begin{array}{l}0.16 \\
0.15\end{array}$ & $\begin{array}{l}215.0_{-128.1}^{+123.1} \\
243.5_{-163.5}^{+167.8}\end{array}$ & 1.04 \\
\hline ARK 564 & pha $(\mathrm{T}+\mathrm{PL}+\mathrm{pcf} * \mathrm{PL}+\mathrm{L}+\mathrm{L})$ & $6.38^{f}$ & $3.42_{-0.12}^{+0.13}$ & $0.97_{-0.04}^{+0.04}$ & & $2.32_{-0.10}^{+0.08}$ & $\begin{array}{l}6.33_{-0.05}^{+0.05} \\
6.71_{-0.03}^{+0.04}\end{array}$ & $\begin{array}{l}0.29 \\
0.41\end{array}$ & $\begin{array}{l}20.9_{-9.9}^{+10.4} \\
32.3_{-11.5}^{+11.4}\end{array}$ & 1.09 \\
\hline NGC 7469 & pha $(\mathrm{T}+\mathrm{abs} * \mathrm{pcf} * \mathrm{PL}+\mathrm{L})$ & $4.96^{f}$ & & $0.18_{-0.01}^{+0.01}$ & & $2.04_{-0.02}^{+0.02}$ & $6.41_{-0.02}^{-0.01}$ & 2.03 & $64.2_{-9.5}^{+7.5}$ & 1.09 \\
\hline MRK 926 & pha(pcf*PL+R+L) & $3.59^{f}$ & & & & $1.98_{-0.02}^{+0.03}$ & $6.33_{-0.06}^{+0.06}$ & 1.24 & $29.1_{-23.8}^{+17.3}$ & 0.96 \\
\hline MRK 530 & pha $(T+p c f * P L+L)$ & $4.06^{f}$ & & $0.20_{-0.02}^{+0.02}$ & & $2.28_{-0.01}^{+0.03}$ & $6.40_{-0.05}^{+0.05}$ & 1.49 & $47.2_{-8.2}^{+7.4}$ & 1.09 \\
\hline MRK 348 & pha $(\mathrm{PL}+$ pha*pcf*PL+L) & $5.91^{f}$ & $2.75_{-0.16}^{+0.17}$ & & $6.86_{-1.01}^{+0.84}$ & $1.70_{-0.06}^{+0.07}$ & $6.40_{-0.04}^{+0.04}$ & 1.59 & $34.2_{-10.4}^{+10.8}$ & 1.05 \\
\hline MRK 1 & $\mathrm{pha}(\mathrm{T}+\mathrm{PL}+\mathrm{R}+\mathrm{L})$ & $5.31^{f}$ & $2.55_{-0.29}^{+0.30}$ & $0.82_{-0.10}^{+0.09}$ & $>150$ & $2.0^{f} \mathrm{R}$ & $6.77_{-0.09}^{+0.08}$ & 0.01 & $1249.2_{-857.5}^{+124.4}$ & 0.99 \\
\hline NGC 2273 & pha $(\mathrm{PL}+\mathrm{R}+\mathrm{L})$ & $7.75_{-7.70}^{+36.20}$ & $2.79_{-0.82}^{+1.87}$ & & $>150$ & $0.67_{-0.92}^{+0.37} \mathrm{R}$ & $6.40_{-0.02}^{+0.03}$ & 2.74 & $2189.2_{-441.7}^{+446.2}$ & 0.97 \\
\hline MRK 78 & pha $(\mathrm{PL}+\mathrm{R}+\mathrm{L})$ & $65.38_{-24.48}^{+30.02}$ & $7.05_{-1.84}^{+1.89}$ & & $>150$ & $1.01_{-0.54}^{+0.44} \mathrm{R}$ & $6.37_{-0.09}^{+0.05}$ & 0.56 & $673.0_{-389.2}^{+402.9}$ & 0.85 \\
\hline NGC $5135^{g}$ & pha $(\mathrm{T}+\mathrm{T}+\mathrm{pha} * \mathrm{PL}+\mathrm{L})$ & $4.58^{f}$ & & $\begin{array}{l}0.08 \\
0.39\end{array}$ & $>150$ & 1.5 & $\begin{array}{l}6.40 \\
1.78\end{array}$ & $\begin{array}{c}0.52 \\
\ldots .\end{array}$ & $\begin{array}{c}1700.0_{-800.0}^{+600.0} \\
\ldots .\end{array}$ & .... \\
\hline MRK $477^{l}$ & pha(pha*PL+L) & $1.30^{f}$ & & & $24.0_{-12.0}^{+17.0}$ & $1.9^{f}$ & $6.40_{-0.23}^{+0.21}$ & $\cdots$ & $560.0_{-500.0}^{+560.0}$ & 0.35 \\
\hline NGC $5929^{c}$ & pha $(\mathrm{PL}+$ pha*PL $+\mathrm{L})$ & 51.60 & 1 & & 27.7 & 1.7 & 6.40 & $\ldots$. & 350.0 & $\ldots$ \\
\hline NGC 7212 & pha $(\mathrm{PL}+\mathrm{R}+\mathrm{L}+\mathrm{L})$ & $5.41^{f}$ & $2.32_{-0.20}^{+0.23}$ & & $>150$ & $0.99_{-0.37}^{+0.54} \mathrm{R}$ & $\begin{array}{l}6.42_{-0.04}^{+0.04} \\
0.89_{-0.04}^{+0.04}\end{array}$ & $\begin{array}{l}0.74 \\
0.04\end{array}$ & $\begin{array}{c}712.3_{-133.4}^{+256.0} \\
59.6_{-38.5}^{+43.1}\end{array}$ & 1.15 \\
\hline MRK 533 & $\operatorname{pha}(\mathrm{T}+\mathrm{PL}+\mathrm{R}+\mathrm{L}+\mathrm{L})$ & $5.16^{f}$ & $3.75_{-0.61}^{+1.45}$ & $0.76_{-0.10}^{+0.13}$ & $>150$ & $2.12_{-0.51}^{+0.49} \mathrm{R}$ & $\begin{array}{l}6.40_{-0.05}^{+0.04} \\
7.04_{-0.04}^{+0.06}\end{array}$ & $\begin{array}{l}0.55 \\
0.53\end{array}$ & $\begin{array}{l}557.2_{-292.7}^{+241.2} \\
665.6_{-333.7}^{+387.9}\end{array}$ & 0.97 \\
\hline NGC 7682 & $\operatorname{pha}(\mathrm{T}+\mathrm{R}+\mathrm{L}+\mathrm{L})$ & $34.88_{-25.11}^{+57.82}$ & $5.36_{-2.38}^{+2.78}$ & & $>150$ & $1.77_{-0.61}^{+0.58} \mathrm{R}$ & $\begin{array}{l}6.43_{-0.06}^{+0.18} \\
7.11_{-0.09}^{+0.39}\end{array}$ & $\begin{array}{l}0.20 \\
0.13\end{array}$ & $\begin{array}{l}477.5_{-312.3}^{+312.9} \\
457.6_{-402.9}^{+460.3}\end{array}$ & 0.81 \\
\hline
\end{tabular}

Notes. ${ }^{(f)}$ Fixed; s: soft component; pha: photoelectric absorption; PL: powerlaw; L: Emission line fitted with a Gaussian; T: Thermal emission from hot gas (MEKAL model in XSPEC); abs: Ionized absorber (ABSORI model in XSPEC); R: Reflection from cold neutral material (PEXRAV model in XSPEC); pcf: partial covering. ${ }^{(g)}$ Guainazzi et al. (2005a); ${ }^{(l)}$ Levenson et al. (2001); ${ }^{(c)}$ Cardamone et al. (2007); emission line fluxes $\left(I_{\text {line }}\right)$ are in units of $10^{-13} \mathrm{erg} \mathrm{cm}^{-2} \mathrm{~s}^{-1}$.

slopes of these two components are the same. We note that for many of our sample sources, the $0.5-10.0 \mathrm{keV} \mathrm{X-ray} \mathrm{spectra}$ can be modeled approximately as " $\exp \left(-N_{\mathrm{H}_{0}} \sigma_{\text {abs }}\right) \times$ [soft component $+\exp \left(-N_{\mathrm{H}_{1}} \sigma_{\mathrm{abs}}\right)\left(\mathrm{AE}^{\Gamma}+\right.$ emission lines $\left.)\right]$ ", as suggested by Nandra \& Pounds (1994); Awaki et al. (2000), where $N_{\mathrm{H}_{0}}$ and $N_{\mathrm{H}_{1}}$ are the Galactic and source column densities, respectively. Table 2 describes the best-fit spectral parameters of all the sample sources and Fig. 1 shows the best spectral fits of 17 sample sources. Errors on the spectral fit parameters and derived quantities are at the $90 \%$ confidence level.

\subsection{X-ray spectral components}

In the following sections we describe the main components of the $0.5-10 \mathrm{keV}$ spectra of our sample Seyfert galaxies.

\subsubsection{Absorbed powerlaw}

The X-ray continuum of Seyfert galaxies can primarily be characterized by a photoelectric absorbed powerlaw. Figure 3 shows the distributions of equivalent hydrogen column density for the two subtypes of Seyferts of our sample. The estimated column densities in Seyfert galaxies of our sample vary from the Galactic value to as high as the limiting value of Compton-thick obscuration $\left(N_{\mathrm{H}} \geq 1.5 \times 10^{24} \mathrm{~cm}^{-2}\right)$. As expected in the unification scheme, Seyfert type $1 \mathrm{~s}$ in our sample are systematically less absorbed than type $2 \mathrm{~s}$.

Among type 2s, many sources (e.g., MRK 1, NGC 2273, MRK 78, NGC 5135, NGC 7212, MRK 533, and NGC 7682) are heavily obscured and show only the reflection component in the $2.0-10 \mathrm{keV}$ band. We identify these seven type 2 Seyferts as Compton-thick sources with equivalent hydrogen column density along the line-of-sight $1.5 \times 10^{24} \mathrm{~cm}^{-2}$ or higher and these exhibit reflection-dominated spectra in the $2.0-10.0 \mathrm{keV}$ band. The hard (2.0-10.0 keV) component of all the Seyfert type 1s is fitted with an absorbed powerlaw and our best fits render photon indices $(\Gamma) \sim 1.5-2.5$ with the mean value of $\sim 2.0$, while type $2 \mathrm{~s}$ have either similar hard X-ray photon indices with high absorption or only a pure reflection component. In those cases where both direct and reflection components are considered, we fix the reflection component photon index equal to that of the direct powerlaw component. In some cases equally good fits can be obtained either with unabsorbed or less absorbed flat $(\Gamma \sim 0.5-1.0)$ powerlaw or with relatively more absorbed steep $(\Gamma \sim 1.8-2.1)$ powerlaw. In such cases we preferred the more absorbed, steep 
V. Singh et al.: X-ray spectral properties of Seyfert galaxies and the unification scheme
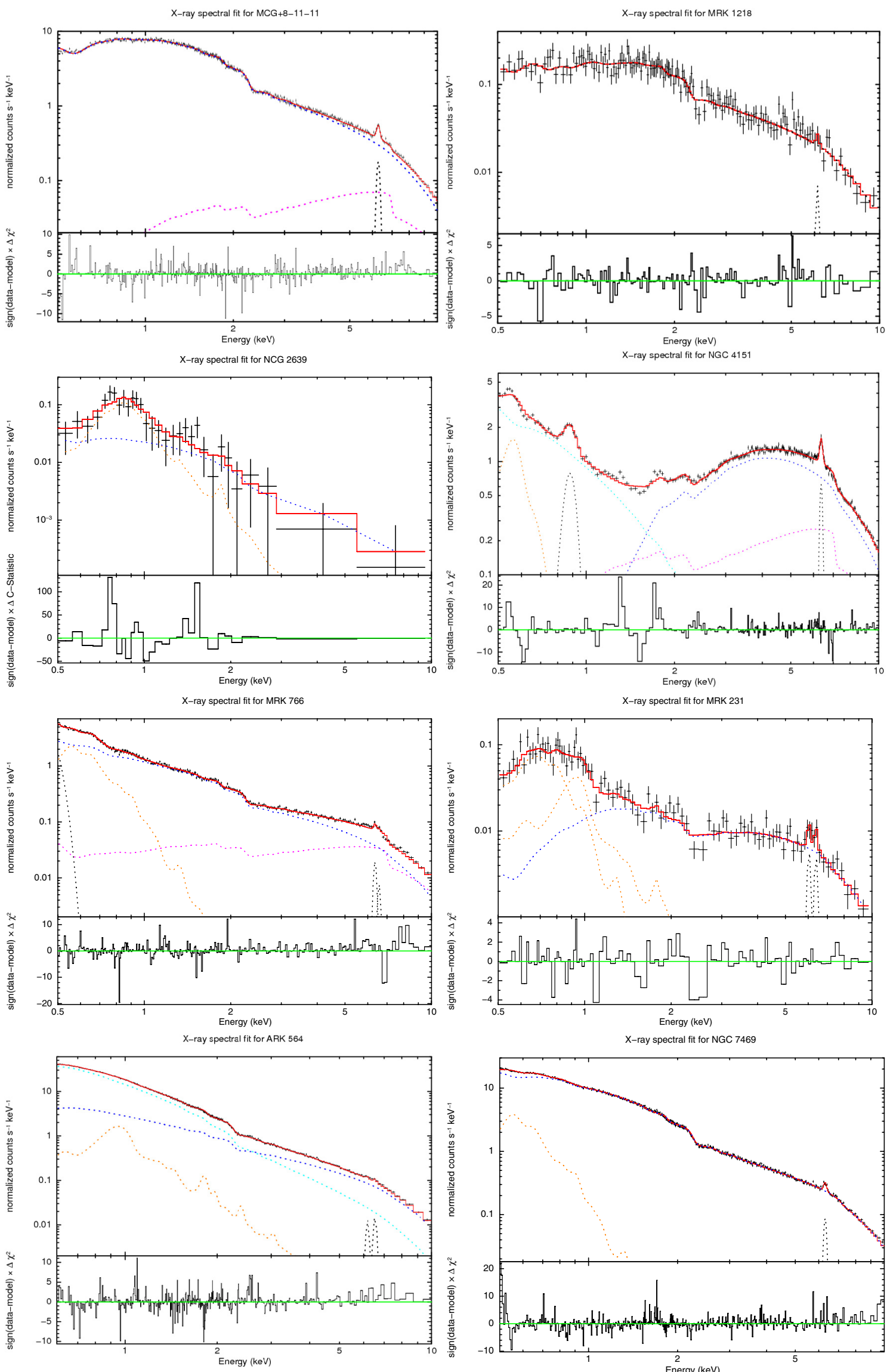

Fig. 1. XMM-Newton pn 0.5-10.0 keV spectral fits for MCG+8-11-11, MRK 1218, NGC 2639, NGC 4151, MRK 766, MRK 231, ARK 564, and NGC 7469. For each source, top panel shows the cumulative fit (solid curve) along with all the additive spectral components (shown as dotted curves) against the spectral data points and bottom panel shows the residuals. 
A\&A 532, A84 (2011)
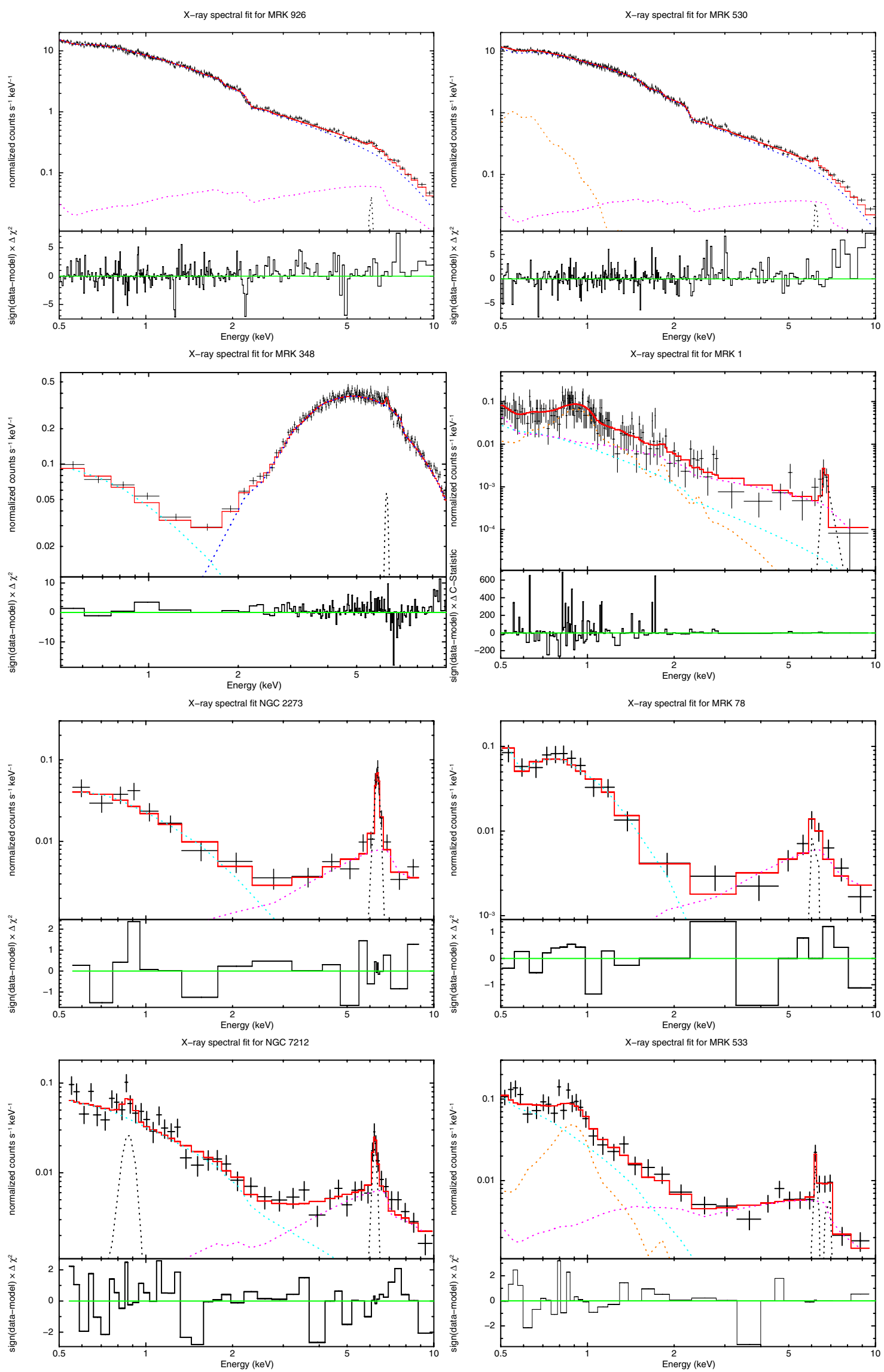

Fig. 1. continued. Spectral fits for MRK 926, MRK 530, MRK 348, MRK 1, NGC 2273, MRK 78, NGC 7212, and MRK 533. 


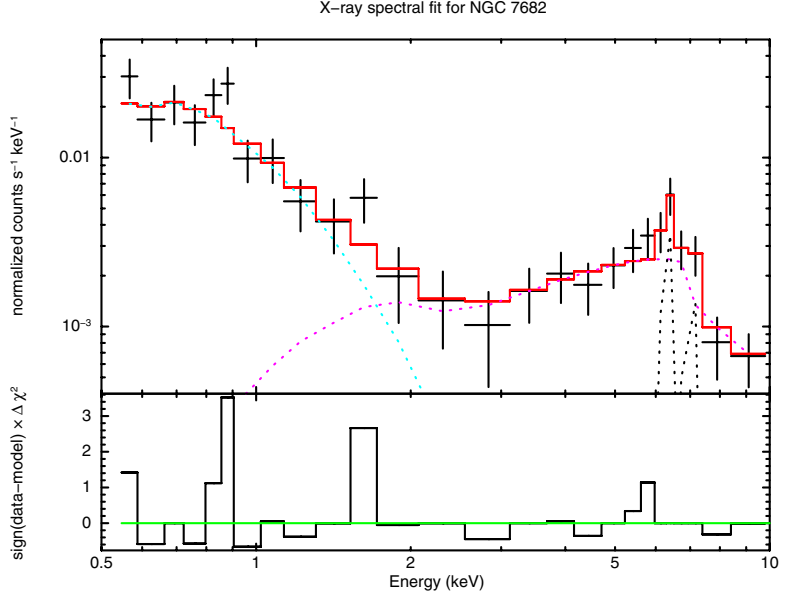

Fig. 1. continued. Spectral fit for NGC 7682.

powerlaw fit because the powerlaw photon index is closer to the canonical value for Seyfert galaxies (Cappi et al. 2006). We did not perform the statistical comparison of the photon indices of the two Seyfert subtypes since in many of the type 2 sources, the 2.0-10.0 keV hard X-ray spectrum is best fitted by a pure reflection component and without any transmitted powerlaw component from AGN. Therefore in such reflection-dominated sources, we do not have the photon index of the direct powerlaw component.

\subsubsection{Soft X-ray excess}

17 among the 20 Seyferts of our sample exhibit the soft excess, i.e., emission above the extrapolation of the absorbed AGN powerlaw. Three (type 1) sources, i.e., MCG+8-11-11, MRK 1218, and MRK 926, do not show any soft excess. We fit the soft excess by a thermal plasma model MEKAL (Mewe-Kaastra-Liedahl 1995) and/or by a steep powerlaw. The typical temperature of the thermal plasma corresponds to $k T \simeq 0.1-1.0 \mathrm{keV}$, and the soft X-ray photon index is typically $2.5-3.5$; however, in two cases (MRK 78, NGC 7682) the best-fit photon index is as steep as $\sim 7.0$ and 5.4, respectively. In some cases (e.g., NGC 4151, MRK 766, and NGC 7212), we see emission line features in 0.5-2.0 keV part of the spectrum, which indicates the presence of a sub-keV thermal plasma.

The origin of soft excess can be attributed to circumnuclear gas heated to a million degrees by shocks induced by AGN outflows (King 2005), an intense circumnuclear star formation (Cid Fernandes et al. 1998; González Delgado et al. 2001), or the photoionization and photoexcitation of circumnuclear gas by the primary AGN emission. Recent high-resolution spectra of a few sources, e.g., NGC 1068 (Young et al. 2001; Kinkhabwala et al. 2002), the Circinus galaxy (Sambruna et al. 2001), MRK 3 (Sako et al. 2000; Bianchi et al. 2005b; Pounds \& Page 2005); NGC 4151 (Schurch et al. 2004) have provided evidence that the soft excess is largely due to circumnuclear gas photoionized by primary AGN emission. Using a large sample of type 1 AGNs, Mateos et al. (2010) noticed soft X-ray excess in a substantial fraction of nearby sources and fitted it with a thermal plasma model with mean rest-frame temperature $k T \sim 100 \mathrm{eV}$. Mateos et al. (2010) ruled out the origin of the soft excess as thermal emission from the accretion disk or Compton-scattered disk emission on the basis of the temperatures detected and the lack of any correlation of the soft excess temperature with the hard X-ray luminosity.

\subsubsection{Reflection component}

Previous X-ray spectral studies report that many Seyferts, in particular type $2 \mathrm{~s}$, show the presence of a reflection component (Awaki et al. 2000). Compton reflection of nuclear radiation from optically thick material can be an important component in Seyfert type 2s, and besides absorption, a flat spectrum can result from the Compton-reflection component (Matt et al. 1996). In highly obscured $\left(N_{\mathrm{H}} \geq 1.5 \times 10^{24} \mathrm{~cm}^{-2}\right)$, i.e., Comptonthick sources, the primary radiation is almost completely absorbed (at $E \leq 10 \mathrm{keV}$ ), but these sources are still observable through the reflected radiation. There are seven type 2 sources in our sample, in which the $2.0-10.0 \mathrm{keV}$ part of the spectrum is best-fitted with a reflection component alone (PEXRAV model in XSPEC; Magdziarz \& Zdziarski 1995). The large $E W$ of Fe $\mathrm{K} \alpha$ line in these sources is consistent with the Compton-thick obscuration and reflection-dominated spectrum (Ghisellini et al. 1994; Levenson et al. 2006). Some type 1 sources, e.g., MCG+8-1111, NGC 4151, MRK 766, and MRK 926 also show a reflection component at the hard end of the spectrum. This can be expected in the framework of the unification scheme since a similar obscuring material having toroidal geometry is present, but the plane of the torus lies away from the line-of-sight, so the reflection component is much fainter compared to the direct transmitted component. Moreover, at the hard end of the spectrum $(E \geq 8.0 \mathrm{keV})$, the reflection component may have a significant contribution.

\subsubsection{Fe $\mathrm{K}_{\alpha}$ and other emission lines}

We detected fluorescent $\mathrm{Fe} \mathrm{K} \alpha$ emission line at $\sim 6.4 \mathrm{keV}$ in all (except NGC 2639) of our sample sources, suggesting the ubiquitous presence of $\mathrm{Fe} \mathrm{K} \alpha$ line in the X-ray spectra of Seyfert galaxies. In most of the sources, the centroid energy of Fe $\mathrm{K} \alpha$ emission line is consistent with the emission from neutral or mildly ionized iron. In some sources, e.g., MRK 766, MRK 231, ARK 564, MRK 533, and NGC 7682, an emission line at $\sim 6.7-7.1 \mathrm{keV}$ is also present, which is possibly either $\mathrm{H}$ or He like ionized $\mathrm{Fe} \mathrm{K} \alpha$ line component or a neutral component of $\mathrm{Fe} \mathrm{K}_{\beta}$ emission line. When the absorbing column density increases to a few times $10^{23} \mathrm{~cm}^{-2}$, the equivalent width of $\mathrm{Fe} \mathrm{K} \alpha$ increases, since it is measured against a depressed continuum. The equivalent width can be higher than $1.0 \mathrm{keV}$ for column densities of $N_{\mathrm{H}} \geq 10^{24} \mathrm{~cm}^{-2}$, and such values are indeed observed in heavily absorbed and Compton-thick sources (Maiolino et al. 1998). Figure 3 shows the distributions of $E W$ s of $\mathrm{Fe} \mathrm{K} \alpha$ line for the two subtypes of Seyferts in our sample. Seyfert type 1s have $E W \mathrm{~s} \sim 20-250 \mathrm{eV}$ while for type 2s (except MRK 348) it ranges from $\sim 0.5 \mathrm{keV}$ to $\sim 2.0 \mathrm{keV}$. High $E W \mathrm{~s}$ of $\mathrm{Fe} \mathrm{K} \alpha$ line in type $2 \mathrm{~s}$ suggests higher obscuration than for type 1s.

Apart from the ubiquitous $\mathrm{Fe} \mathrm{K} \alpha$ emission line, we also notice other emission lines in some of our sample sources, mainly in the soft X-ray band. The addition of a Gaussian emission line at $\sim 0.88 \mathrm{keV}$ in NGC 4151 and NGC 7212 improves the fit and is likely to be associated with $\mathrm{O}$ VIII radiative recombination continua (Griffiths et al. 1998). High-resolution spectroscopic observations show that the soft X-ray emission in NGC 4151 is dominated by X-ray emission lines and radiative recombination continua (RRC) from hydrogen-like and helium-like ionization states of neon, oxygen, nitrogen, and carbon (Schurch et al. 2004). Also, in MRK 766 the addition of a Gaussian emission feature at $\sim 0.5 \mathrm{keV}$ improves the fit and may arise from the blending of few narrow emission lines. 

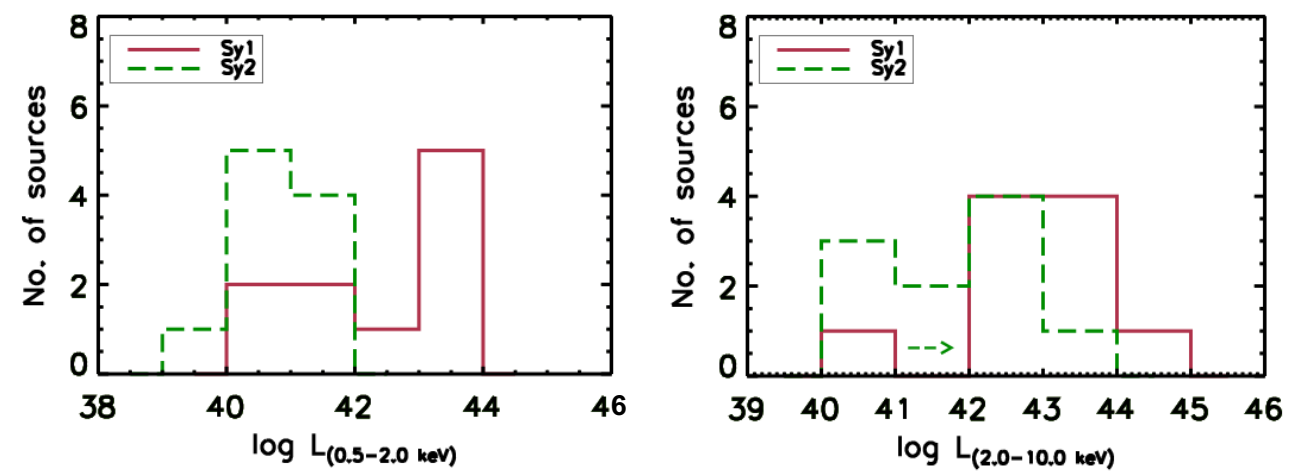

Fig. 2. Distributions of the soft X-ray (0.5-2.0) and hard X-ray (2.0-10.0) luminosities for the two subtypes of Seyfert galaxies.
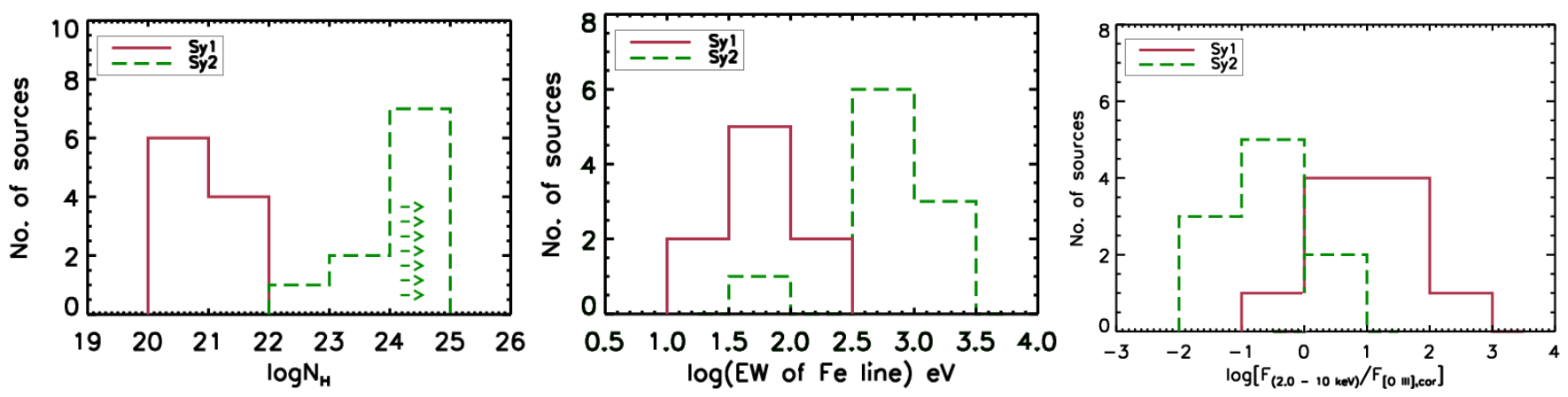

Fig. 3. Distributions of the equivalent hydrogen column density, the equivalent width of Fe $\mathrm{K} \alpha$, and the flux ratio of hard X-ray to [OIII] $\lambda 5007 \AA$ line for the two subtypes of Seyfert galaxies.

\section{Comparison of the X-ray spectral properties of the two Seyfert subtypes}

In this section we discuss the statistical comparison of the distributions of X-ray luminosities and spectral properties of the two subtypes of Seyferts in the framework of Seyfert unification scheme.

\subsection{Soft $(0.5-2.0 \mathrm{keV})$ and hard $(2.0-10.0 \mathrm{keV})$ band $X$-ray luminosities}

Table 5 lists the fluxes, luminosities in both the soft $(0.5-$ $2.0 \mathrm{keV})$ and hard (2.0-10.0 keV) X-ray bands, and the flux ratios of the hard X-ray to [OIII] $\lambda 5007 \AA$ Al line emission. Figure 2 shows the X-ray luminosity distributions for the two Seyfert subtypes in the soft $(0.5-2.0 \mathrm{keV})$ and hard $(2.0-10.0 \mathrm{keV}) \mathrm{X}$-ray bands. The observed soft X-ray luminosities for Seyfert type 1s in our sample range from $\sim 10^{40} \mathrm{erg} \mathrm{s}^{-1}$ to $\sim 10^{44} \mathrm{erg} \mathrm{s}^{-1}$ with the median value $\sim 6.58 \times 10^{42} \mathrm{erg} \mathrm{s}^{-1}$, while for Seyfert type $2 \mathrm{~s}$ it ranges from $\sim 10^{38} \mathrm{erg} \mathrm{s}^{-1}$ to $\sim 10^{42} \mathrm{erg} \mathrm{s}^{-1}$ with the median value $\sim 6.9 \times 10^{40} \mathrm{erg} \mathrm{s}^{-1}$. The two sample Kolmogorov - Smirnov test shows that the soft $(0.5-2.0 \mathrm{keV}) \mathrm{X}$-ray luminosity distributions of type 1 and type 2 Seyferts are completely different; i.e., the null hypothesis that the two distributions are the same is rejected at $99 \%$ level of significance. Seyfert type $2 \mathrm{~s}$ have systematically lower soft X-ray luminosities than type $1 \mathrm{~s}$. The nearly 2 Dex difference in the medians of the soft X-ray luminosities of two Seyfert subtypes can be explained as the presence of more line-of-sight obscuration in type $2 \mathrm{~s}$ than in type $1 \mathrm{~s}$, which is expected since torus intercepts the observer's line-ofsight according to the orientation-based Seyfert unification. In the hard X-ray (2.0-10.0 keV) band, Seyfert 1s of our sample have luminosities ranging from $\sim 10^{40} \mathrm{erg} \mathrm{s}^{-1}$ to $\sim 10^{45} \mathrm{erg} \mathrm{s}^{-1}$ with the median value $\sim 1.1 \times 10^{43} \mathrm{erg} \mathrm{s}^{-1}$, while for Seyfert $2 \mathrm{~s}$ it ranges from $\sim 10^{40} \mathrm{erg} \mathrm{s}^{-1}$ to $\sim 10^{44} \mathrm{erg} \mathrm{s}^{-1}$ with the median value $\sim 6.8 \times 10^{41} \mathrm{erg} \mathrm{s}^{-1}$. The difference in the hard X-ray luminosity distributions of the two Seyfert subtypes is lower than in the soft band and can be explained as hard X-ray (2.0-10.0 keV) photons suffer less absorption. According to the unification model, at high enough energy, one can expect similar X-ray luminosities for both Seyfert subtypes because the torus optical thickness decreases with the increase in photon energy and similar central engines are at work in both subtypes. The substantial difference in the hard X-ray (2.0-10.0 keV) luminosity distributions of the two Seyfert subtypes can be understood as the result of high absorption in type $2 \mathrm{~s}$. We have several heavily obscured (Compton-thick) type 2 sources in our sample in which X-ray continuum below $10.0 \mathrm{keV}$ is completely suppressed and dominated by reprocessed components.

\subsection{Line-of-sight obscuration}

The cold matter present between the observer and the AGN (consisting of the Galactic and the material at the redshift of AGN) mainly absorbs soft X-ray from AGN. The photoelectric absorption component in the X-ray spectral modeling renders the amount of absorption along the line-of-sight in terms of the equivalent hydrogen column density, as long as the direct transmitted component is seen. In general, we fit the X-ray continuum with a photoelectric absorbed powerlaw and keeping column density and powerlaw photon index as free parameters, and also all the spectral components are at least absorbed by the Galactic equivalent hydrogen column density. Figure 3 shows the distributions of cumulative $N_{\mathrm{H}}$ for the two Seyfert subtypes. The estimated absorbing equivalent hydrogen column densities for our sample sources vary from as low as the Galactic 
Table 3. Summary of partial covering model parameters.

\begin{tabular}{lcc}
\hline \hline $\begin{array}{l}\text { Source } \\
\text { name }\end{array}$ & $\begin{array}{c}N_{\mathrm{H}} \\
\left(10^{22} \mathrm{~cm}^{-2}\right)\end{array}$ & $\begin{array}{c}\text { Covering } \\
\text { fraction }(f)\end{array}$ \\
\hline NGC 4151 & $9.06_{-0.98}^{+1.27}$ & $0.69_{-0.05}^{+0.04}$ \\
MRK 766 & $6.42_{-0.56}^{+0.93}$ & $0.51_{-0.03}^{+0.02}$ \\
MRK 231 & $8.35_{-4.64}^{+4.02}$ & $0.74_{-0.25}^{+0.09}$ \\
ARK 564 & $3.09_{-0.45}^{+0.52}$ & $0.61_{-0.15}^{+0.26}$ \\
NGC 7469 & $35.55_{-6.77}^{+9.93}$ & $0.28_{-0.03}^{+0.03}$ \\
MRK 926 & $4.35_{-1.99}^{+2.86}$ & $0.15_{-0.06}^{+0.06}$ \\
MRK 530 & $14.81_{-4.18}^{+5.62}$ & $0.27_{-0.27}^{+0.27}$ \\
MRK 348 & $10.47_{-1.00}^{+0.68}$ & $0.84_{-0.06}^{+0.06}$ \\
\hline
\end{tabular}

Table 4. Summary of ionized absorber parameters.

\begin{tabular}{lcc}
\hline \hline $\begin{array}{l}\text { Source } \\
\text { name }\end{array}$ & $\begin{array}{c}N_{\mathrm{H}} \\
\left(10^{22} \mathrm{~cm}^{-2}\right)\end{array}$ & $\begin{array}{c}\text { Ionization } \\
\text { parameter }(\xi)\end{array}$ \\
\hline MRK 1218 & $0.63_{-0.32}^{+0.41}$ & $39.60_{-38.37}^{+5.40}$ \\
NGC 4151 & $5.08_{-1.20}^{+1.45}$ & $0.31_{-0.28}^{+0.40}$ \\
MRK 766 & $0.21_{-0.02}^{+0.03}$ & $6.61_{-1.67}^{+4.67}$ \\
NGC 7469 & $1.06_{-0.36}^{+0.42}$ & $1193.19_{-232.63}^{+692.30}$ \\
\hline
\end{tabular}

value $\left(N_{\mathrm{H}} \simeq 10^{20} \mathrm{~cm}^{-2}\right)$ to as high as the Compton-thick limiting value $\left(N_{\mathrm{H}} \geq 10^{24} \mathrm{~cm}^{-2}\right)$. For type $1 \mathrm{~s}$, the equivalent $N_{\mathrm{H}}$ ranges from $\sim 10^{20} \mathrm{~cm}^{-2}$ (Galactic value) to $\sim 10^{22} \mathrm{~cm}^{-2}$ with the median value $\sim 10^{21} \mathrm{~cm}^{-2}$, while for type $2 \mathrm{~s}$ it ranges from $\sim 10^{22} \mathrm{~cm}^{-2}$ to $\geq 10^{24} \mathrm{~cm}^{-2}$ with the median value $\geq 10^{24} \mathrm{~cm}^{-2}$. We have $\sim 7 / 10$ reflection-dominated type 2 sources in which the $2.0-10.0 \mathrm{keV}$ hard component is accounted for by a pure reflection component (PEXRAV model), therefore we have only an upper limit of $N_{\mathrm{H}}$ values $\left(\geq 1.5 \times 10^{24}\right)$ in such cases. Seyfert type 2 s have systematically higher absorbing column densities than types $1 \mathrm{~s}$ and the distributions of the absorbing column densities $\left(N_{\mathrm{H}}\right)$ are consistent with the orientation-based unification scheme. In our small sample, we do not find any unobscured type 2 Seyfert, although some previous studies report $10 \%-30 \%$ of unobscured type 2 Seyferts (Panessa \& Bassani 2002). While comparing the amount of obscuration for the two Seyfert subtypes, we considered only cold absorption, which is accounted for by the photoelectric absorption component. In several sources we require partial covering of the primary AGN radiation. The best-fit values of covering fraction and absorbing equivalent hydrogen column density are mentioned in Table 3 . In four Seyfert type 1s, i.e., MRK 1218, NGC 4151, MRK 766, and NGC 7469, warm absorption characterized by the ABSORI model (ionized absorber) is needed for the best fit. While using the ABSORI model, we fixed the photon index of the powerlaw of the absori component equal to the photon index of the primary powerlaw component, and the temperature was fixed to $3 \times 10^{4} \mathrm{~K}$. The best-fit parameter values for $N_{\mathrm{H}}$ and ionization parameters $(\xi)$ are mentioned in Table 4 . The ionization parameter indicates the ionization state of absorbing material and is defined as $\xi=\frac{L}{n r^{2}}$, where $L$ is the source luminosity (in $\sim 13.6 \mathrm{eV}-$ $13.6 \mathrm{keV}), n$ the absorbing gas density, and $r$ the distance of the absorber from the ionizing source (see; Osterbrock \& Ferland 2006). The estimated ionization parameter reveals low ionizing material in MRK 1218, NGC 4151, and MRK 766; however, in NGC 7469, the ionization level is high.

Using XMM-Newton RGS data, Blustin et al. (2007) report that NGC 7469 shows evidence of ionized outflowing material with a wide range of ionization $(\log \xi \sim 0.5-3.5)$. The presence of the warm absorber in NGC 4151 and MRK 766 has also been reported in previous studies (Schurch et al. 2004; Mason et al. 2003).

\subsection{Equivalent width of $\mathrm{Fe} \mathrm{K} \alpha$ line}

We detect fluorescent $\mathrm{Fe} \mathrm{K} \alpha$ emission line in all but one (NGC 2639) of our sample sources suggesting the ubiquitous presence of $\mathrm{Fe} \mathrm{K} \alpha$ line in the X-ray spectra of Seyfert galaxies. Due to the lack of enough counts above $2.0 \mathrm{keV}$ we could not confirm the presence of Fe K $\alpha$ emission line in NGC 2639. However, using ASCA observations of NGC 2639, Terashima et al. (2002) report the presence of an $\mathrm{Fe} \mathrm{K} \alpha$ line with $E W \sim$ 1.5-3.0 keV. We estimated the $E W$ of $\mathrm{Fe} \mathrm{K} \alpha$ line with respect to the modeled continuum and find that Seyfert type $2 \mathrm{~s}$ have systematically larger $E W \mathrm{~s}(\sim 350 \mathrm{eV}$ to $2.2 \mathrm{keV}$, except of MRK 348), with the median value $\sim 600 \mathrm{eV}$ than that of type $1 \mathrm{~s}$ ( $\sim 20-200 \mathrm{eV})$ with the median value $\sim 64.0 \mathrm{eV}$. The two sample Kolmogorov - Smirnov test shows that the distributions of $E W \mathrm{~s}$ of Fe $\mathrm{K} \alpha$ line of the two Seyfert subtypes are completely different (cf., Table 6). Heavily obscured, i.e., Compton-thick sources, are characterized by large $E W$ of the $\mathrm{Fe} \mathrm{K} \alpha$ line (Krolik et al. 1994; Levenson et al. 2006), so we use the large $E W(\sim 1.0 \mathrm{keV})$ of $\mathrm{Fe} \mathrm{K} \alpha$ line because an indicator of heavy obscuration as it is measured against much depressed continuum $\left(N_{\mathrm{H}} \sim 10^{23}-\right.$ $10^{24} \mathrm{~cm}^{-2}$; Leahy \& Creighton 1993) or against pure reflection component $\left(N_{\mathrm{H}} \sim 10^{24}-10^{25}\right.$; Bassani et al. 1999). However, identifying a source as "Compton-thick" only on the basis of the large $E W$ of $\mathrm{Fe} \mathrm{K} \alpha$ line may not be correct, since a large $E W$ can arise if the ionizing radiation is anisotropic (Ghisellini et al. 1991), or if there is a lag between a drop in the continuum and line emissions, as observed in NGC 2992 by Weaver et al. (1996).

\subsection{Flux ratio of hard X-ray to [OIII] $\lambda 5007 \AA$}

In order to confirm the higher obscuration in Seyfert type $2 \mathrm{~s}$ than in type $1 \mathrm{~s}$, we used the flux ratio of hard (2.0-10.0 keV) X-ray to [OIII] $\lambda 5007 \AA$ and the equivalent width of $\mathrm{Fe} \mathrm{K} \alpha$ line as diagnostic tools. Bassani et al. (1999) report that the flux ratio of hard X-ray to [OIII] $\lambda 5007 \AA$ can be used effectively in identifying Compton-thin and Compton-thick sources with the latter having the flux ratio $\leq 1$. The flux ratio of hard X-ray to [OIII] $\lambda 5007 \AA$ can be used to indicate the amount of obscuration since [OIII] originates in the NLR region largely unaffected by the torus obscuration and can be considered as the proxy of the intrinsic AGN power, since it is produced by photoionization of NLR clouds by the AGN continuum (Yee et al. 1981; Nelson $\&$ Whittle 1995). While estimating the flux ratio of hard X-ray to [OIII] $\lambda 5007 \AA$, the latter were corrected for the optical reddening whenever the Balmer decrement value was available for a source in the literature. We have Balmer decrement $\left(\mathrm{H}_{\alpha} / \mathrm{H}_{\beta}\right)$ values, hence, optical reddening corrected [OIII] fluxes for only 16 (6 type $1 \mathrm{~s}$ and 10 type $2 \mathrm{~s}$ ) sources in our sample. For reddening correction, we used the formula given in Bassani et al. (1999), i.e., $F_{[\mathrm{OIII}] \text {, cor }}=F_{[\mathrm{OIII}] \text {, obs }}\left[\left(\mathrm{H}_{\alpha} / \mathrm{H}_{\beta}\right) /\left(\mathrm{H}_{\alpha} / \mathrm{H}_{\beta} \mathrm{S}\right)_{0}\right]^{2.94}$, where the intrinsic Balmer decrement $\left(\mathrm{H}_{\alpha} / \mathrm{H}_{\beta}\right)_{0}$ is assumed to be 3.0, and only narrow components of $\mathrm{H}_{\alpha}$, and $\mathrm{H}_{\beta}$ are considered.

The statistical comparison of the distributions of the flux ratios of hard X-ray $(2.0-10.0 \mathrm{keV})$ to [OIII] for the two Seyfert subtypes again confirms the systematically higher obscuration is type $2 \mathrm{~s}$ than type $1 \mathrm{~s}$ (cf., Table 6, Fig. 3). Also, using this flux ratio as an indicator, we note that MRK 1, MRK 78, NGC 5135, MRK 477, NGC 7212, MRK 533, and NGC 7682 have Compton-thick obscuration. X-ray spectral properties, e.g., reflection-dominated hard X-ray component, 
A\&A 532, A84 (2011)

Table 5. Observed X-ray fluxes and luminosities in the soft $(0.5-2.0 \mathrm{keV})$ and hard (2.0-10.0 keV) bands and the flux ratios of hard X-ray to [OIII].

\begin{tabular}{lcccccccccc}
\hline \hline Name & $F_{0.5-2.0 \mathrm{keV}}$ & $\log L_{0.5-2.0 \mathrm{keV}}$ & $F_{2.0-10 \mathrm{keV}}$ & $\log L_{2.0-10 \mathrm{keV}}$ & $F_{[\text {[OII] }}^{\text {obs. }}$ & Ref. & $\mathrm{H}_{\alpha} / \mathrm{H}_{\beta}$ & Ref. & $F_{[\mathrm{OIII]}]}^{\text {cor }}$ & $\log R_{\mathrm{x}}$ \\
\hline MCG+8-11-11 & 161.18 & 43.35 & 441.97 & 43.79 & 6.43 & 1 & 4.37 & 7 & 19.43 & 1.36 \\
MRK 1218 & 4.86 & 41.96 & 25.35 & 42.68 & 1.70 & 2 & 3.26 & 8 & 2.17 & 1.07 \\
NGC 2639 & 1.26 & 40.52 & 0.75 & 40.29 & 1.07 & 3 & 4.16 & 9 & 2.79 & -0.57 \\
NGC 4151 & 29.49 & 40.75 & 828.83 & 42.20 & 116.00 & 4 & 3.1 & 7 & 127.74 & 0.81 \\
MRK 766 & 33.99 & 42.10 & 72.66 & 42.43 & 3.95 & 1 & 5.1 & 7 & 18.79 & 0.59 \\
MRK 231 & 0.99 & 41.60 & 6.06 & 42.38 & 2.30 & 2 & $\ldots$ & $\ldots$ & $\ldots$ & 0.42 \\
ARK 564 & 364.22 & 43.67 & 162.25 & 43.31 & 1.90 & 4 & $\ldots$ & $\ldots$ & $\ldots$ & 1.93 \\
NGC 7469 & 213.27 & 43.08 & 293.41 & 43.21 & 5.80 & 4 & 9.33 & 7 & 162.98 & 0.26 \\
MRK 926 & 171.95 & 43.94 & 299.05 & 44.18 & 3.50 & 4 & $\ldots$ & $\ldots$ & $\ldots$ & 1.93 \\
MRK 530 & 166.48 & 43.52 & 182.74 & 43.56 & 0.48 & 4 & $\ldots$ & $\ldots$ & $\ldots$ & 2.58 \\
MRK 348 & 1.17 & 40.76 & 269.39 & 43.12 & 4.12 & 1 & 6.02 & 7 & 31.93 & 0.93 \\
MRK 1 & 0.98 & 40.74 & 0.81 & 40.66 & 6.00 & 4 & 5.89 & 7 & 43.61 & -1.73 \\
NGC 2273 & 0.53 & 39.62 & 10.02 & 40.89 & 1.60 & 5 & 6.92 & 7 & 18.68 & -0.27 \\
MRK 78 & 0.79 & 41.39 & 5.52 & 42.23 & 6.60 & 4 & 6.46 & 7 & 62.93 & -1.06 \\
NGC 5135 & 1.90 & 40.91 & 1.60 & 40.83 & 3.70 & 6 & 7.8 & 9 & 61.66 & -1.59 \\
MRK 477 & 1.20 & 41.59 & 12.0 & 42.59 & 15.00 & 4 & 5.4 & 7 & 84.45 & -0.85 \\
NGC 5929 & 0.81 & 40.15 & $>14.0$ & $>41.39$ & 0.93 & 4 & 5.5 & 7 & 5.53 & $>0.40$ \\
NGC 7212 & 0.83 & 41.13 & 6.96 & 42.05 & 8.75 & 1 & 5.01 & 7 & 39.52 & -0.75 \\
MRK 533 & 1.42 & 41.42 & 6.08 & 42.06 & 5.21 & 1 & 5.0 & 7 & 23.39 & -0.59 \\
NGC 7682 & 0.27 & 40.22 & 2.62 & 41.22 & 2.30 & 4 & 4.8 & 7 & 9.16 & -0.54 \\
\hline
\end{tabular}

Notes. [OIII] and X-ray fluxes are in unit of $10^{-13} \mathrm{erg} \mathrm{cm}^{-2} \mathrm{~s}^{-1} \cdot R_{\mathrm{x}}=\frac{\text { Flux }(2.0-10.0 \mathrm{keV})}{\text { Flux[OIII] }}{ }^{(g)}$ Guainazzi et al. (2005a); ${ }^{(l)}$ Levenson et al. (2001); ${ }^{(c)}$ Cardamone et al. (2007).

References. 1: Schmitt et al. (2003); 2: Dahari \& De Robertis (1988); 3: Panessa et al. (2006); 4: Whittle (1992); 5: Ferruit et al. (2000); 6: Polletta et al. (1996); 7: Mulchaey et al. (1994); 8: Dahari \& De Robertis (1988); 9: Bassani et al. (1999).

Table 6. Medians and two sample Kolmogorov - Smirnov tests for statistical comparison of various distributions of the two Seyfert subtypes.

\begin{tabular}{|c|c|c|c|c|}
\hline \multirow{2}{*}{ Distribution } & \multicolumn{2}{|c|}{ Median } & \multirow[t]{2}{*}{$D$} & \multirow[t]{2}{*}{$p$-value } \\
\hline & Type $1 \mathrm{~s}$ & Type $2 \mathrm{~s}$ & & \\
\hline $\log L_{0.5-2.0 \mathrm{keV}}$ & 42.82 & 40.84 & 0.8 & $2.06 \times 10^{-3}$ \\
\hline $\log L_{2.0-10.0 \mathrm{keV}}$ & 43.02 & 41.83 & 0.6 & $5.25 \times 10^{-2}$ \\
\hline $\log N_{\mathrm{H}}$ & 21.02 & $>24.18$ & 1.0 & $9.08 \times 10^{-5}$ \\
\hline $\log E W(\mathrm{eV})$ & 1.81 & 2.79 & 0.9 & $9.30 \times 10^{-4}$ \\
\hline $\log R_{\mathrm{X}}$ & 0.96 & -0.66 & 0.7 & $1.48 \times 10^{-2}$ \\
\hline
\end{tabular}

Notes. The two sample Kolmogorov - Smirnov test examines the hypothesis that two samples come from same distribution. $D=$ $\operatorname{Sup} x|\mathrm{~S} 1(x)-\mathrm{S} 2(x)|$ is the maximum difference between the cumulative distributions of two samples $\mathrm{S} 1(x)$ and $\mathrm{S} 2(x)$, respectively. $p$-value is the probability that the null hypothesis, i.e., two samples comes from same distribution, is correct.

low hard X-ray to [OIII] flux ratio and high $E W$ of $\mathrm{Fe} \mathrm{K} \alpha$ line implies that $\sim 7-8$ out of 10 type 2 sources of our sample are Compton-thick $\left(N_{\mathrm{H}} \geq 1.5 \times 10^{24} \mathrm{~cm}^{-2}\right)$. Some of our sample sources, e.g., MRK 477 (Bassani et al. 1999), NGC 5135, NGC 7212 (Levenson et al. 2006), NGC 2273 (Awaki et al. 2009), and MRK 533 (Bianchi et al. 2005a) have already been reported as Compton-thick. In our sample we have only one Seyfert subtype 1.8 (MRK 1218) and one subtype 1.9 (NGC 2639) which are included in type 1s. Even if we include subtypes $1.8,1.9$ in type $2 \mathrm{~s}$, the relative fraction of Compton-thick sources thus remains high $\sim 60 \%$ (7-8 source out of 12 type $2 \mathrm{~s}$ ).

\section{Conclusions}

We investigated the $0.5-10 \mathrm{keV}$ X-ray spectral properties of a sample of 20 Seyfert galaxies in which the two Seyfert subtypes have matched distributions in orientation-independent parameters. We summarize the conclusions of our study below.

- The X-ray spectra of our sample Seyfert galaxies, in general, are best fitted with a model that consists of: (i) a powerlaw with a photoelectric cutoff due to the cold absorption from the Galactic column density and the neutral gas at the redshift of the AGN; (ii) a narrow Gaussian fitted to the Fe $\mathrm{K} \alpha$ fluorescent emission line at $6.4 \mathrm{keV}$; (iii) an often-seen soft excess component characterized by either a steep powerlaw and/or a thermal plasma model with temperature $k T \sim 0.1-$ $1.0 \mathrm{keV}$.

- In several of the heavily obscured type 2 sources, X-ray spectra are completely dominated by the reflection component, and the hard part of spectra can be characterized with the PEXRAV model and a prominent $\mathrm{Fe} \mathrm{K} \alpha$ line.

- Seyfert type 1s systematically have higher observed soft $\mathrm{X}$-ray luminosities than type $2 \mathrm{~s}$, as would be expected in the unification scheme with the hypothesis that in type $2 \mathrm{~s}$, an obscuring torus intercepts the AGN view and therefore absorbs the soft X-ray photons emanating from the AGN. The distributions of the observed hard $(2.0-10 \mathrm{keV}) \mathrm{X}$-ray luminosities of the two subtypes differ much less significantly, which is consistent with the prediction of unification, because the optical thickness of obscuring material decreases with the increase in X-ray photons energy.

- The X-ray absorbing column density for Seyfert type 1s in our sample ranges from $\sim 10^{20} \mathrm{~cm}^{-2}$ (Galactic value) to $\sim 10^{22} \mathrm{~cm}^{-2}$ with the median value $\sim 10^{21} \mathrm{~cm}^{-2}$, while for type $2 \mathrm{~s}$ it ranges from $\sim 10^{22} \mathrm{~cm}^{-2}$ to Compton-thick limiting value $\geq 10^{24} \mathrm{~cm}^{-2}$ with the median value $\geq 10^{24} \mathrm{~cm}^{-2}$. The distribution of X-ray absorbing column densities $\left(N_{\mathrm{H}}\right)$ for Seyfert galaxies in our sample is broadly consistent with the previous studies (e.g., Cappi et al. 2006; Akylas \& Georgantopoulos 2009). 
- The Fe $\mathrm{K} \alpha$ fluorescent emission line has been detected in all (except one) of our sample sources, suggesting the ubiquitous presence of $\mathrm{Fe} \mathrm{K} \alpha$ line in the X-ray spectra of Seyfert galaxies. The centroid energy $\sim 6.4 \mathrm{keV}$ of the line is consistent with the emission from neutral or mildly ionized iron. The equivalent width of $\mathrm{Fe} \mathrm{K} \alpha$ line in type 2 s ranges $\sim 350 \mathrm{eV}$ to $2.2 \mathrm{keV}$ and is systematically higher than that of $1 \mathrm{~s}(\sim 20-200 \mathrm{eV})$.

- The statistical comparison of X-ray spectral properties: (i) the X-ray absorbing column densities; (ii) the hard X-ray spectral shape (absorbed powerlaw versus reflection dominated); (iii) the $E W \mathrm{~s}$ of $\mathrm{Fe} \mathrm{K} \alpha$ line; (iv) the flux ratios of hard X-ray to [OIII] $\lambda 5007 \AA$; and (v) the luminosity distributions in soft and hard X-ray bands for the two subtypes of Seyfert galaxies, are consistent with the orientation and obscuration-based Seyfert unification scheme.

- We argued for the importance of sample selection in testing the Seyfert unification scheme. And, using a sample based on the properties independent of the orientation of the obscuring torus, AGN, and host galaxy, we show that the $0.5-10 \mathrm{keV}$ $\mathrm{X}$-ray spectral properties are consistent with the unification scheme.

- We also note a high fraction, $\sim 70 \%$, of heavily absorbed likely Compton-thick sources among the type 2 Seyfert population. This high fraction agrees with the previous study by Risaliti et al. (1999) based on an [OIII] 15007 A luminosityselected sample of Seyfert $2 \mathrm{~s}$ and implies that an unbiased sample is essential for estimating the accurate relative fraction of the heavily absorbed, i.e., Compton-thick AGNs. Also, the heavily obscured AGNs may in part be accountable for the missing sources that contribute to the cosmic X-ray background (Worsley et al. 2005; Gilli et al. 2007).

Acknowledgements. This work is based on observations with XMM-Newton, an ESA science mission with instruments and contributions directly funded by ESA Member States and the USA (NASA). Also, this research made use of the NASA/IPAC Extragalactic Database (NED), which is operated by the Jet Propulsion Laboratory, California Institute of Technology, under contract with the National Aeronautics and Space Administration. Authors thank the anonymous referee for useful comments and suggestions for improving the manuscript.

\section{Appendix A: Notes on individual sources}

In this section we describe the X-ray spectral properties of individual sources of our sample and compare of their X-ray spectral properties with the previous studies.

\section{$M C G+8-11-11$}

MCG+8-11-11 has been observed by all the major X-ray satellites, with the exception of Chandra. The ASCA (Grandi et al. 1998) and BeppoSAX (Perola et al. 2000) data were well-fitted by a fairly standard model composed of a powerlaw, a warm absorber, a Compton reflection component, and an Fe $\mathrm{K} \alpha$ line. Matt et al. (2006) fitted the XMM-Newton EPIC pn spectrum with a model composed of an absorbed powerlaw $\left(N_{\mathrm{H}} \simeq\right.$ $\left.1.83_{-0.03}^{+0.06} \times 10^{21} \mathrm{~cm}^{-2}, \Gamma \simeq 1.805 \pm 0.015\right)$, a Compton reflection component (with the inclination angle kept fixed to $30^{\circ}$ ), a Gaussian $\mathrm{Fe} \mathrm{K} \alpha$ line $(E W \sim 75 \pm 15 \mathrm{eV})$, warm absorption $\left(N_{\mathrm{H} \text {,warm }} \simeq 1.1_{-0.06}^{+0.06} \times 10^{22} \mathrm{~cm}^{-2}\right)$ with the temperature of the material fixed at $10^{6} \mathrm{~K}$, and an absorption edge at $0.74 \mathrm{keV}$ corresponding to He-like oxygen. Our best-fit spectral parameters are broadly consistent with Matt et al. (2006), although we get a good fit without using warm absorption. We also confirm the absence of a soft excess in this source using the XMM-Newton EPIC pn data.

\section{MRK 1218}

MRK 1218 does not have Chandra and ASCA observations but has been detected by RoSAT. We present the XMM-Newton spectrum of MRK 1218 for the first time. We find that an absorbed powerlaw $\left(N_{\mathrm{H}} \simeq 1.52_{-1.30}^{+0.43} \times 10^{21} \mathrm{~cm}^{-2}, \Gamma \simeq 1.48_{-0.17}^{+0.11}\right)$, with warm absorption $\left(N_{\mathrm{H} \text {,warm }} \simeq 0.63_{-0.32}^{+0.41} \times 10^{22} \mathrm{~cm}^{-2}\right)$ having plasma temperature fixed at $3 \times 10^{4} \mathrm{~K}$ and an $\mathrm{Fe} \mathrm{K} \alpha$ line of $E W \simeq 63.9 \mathrm{eV}$ gives the best fit.

\section{NGC 2639}

Terashima et al. (2002) fitted the ASCA spectrum of NGC 2639 by a partially covered powerlaw (with $N_{\mathrm{H}, 1} \sim 0.08$ $(\leq 0.32) \times 10^{22} \mathrm{~cm}^{-2}, N_{\mathrm{H}, 2} \sim 32_{-30}^{+12.5} \times 10^{22} \mathrm{~cm}^{-2}$, covering fraction $\sim 0.89_{-0.81}^{+0.08}, \Gamma \sim 2.8_{-0.6}^{+1.0}$, and $\chi^{2} /$ d.o.f. $\simeq 45.8 / 49$ ). They also attempted a model consisting of an absorbed powerlaw plus a Raymond-Smith (Raymond \& Smith 1977) thermal plasma modified by the Galactic absorption. The best-fit parameters reported by Terashima et al. (2002) using this model are $N_{\mathrm{H}, 1} \simeq$ $0.027 \times 10^{22} \mathrm{~cm}^{-2}$ (fixed), $k T \simeq 0.80_{-0.40}^{+0.27}$, abundances fixed to 0.5 of solar, $N_{\mathrm{H}, 2} \sim 0.0(\leq 0.31) \times 10^{22} \mathrm{~cm}^{-2}, \Gamma \simeq 1.92_{-0.37}^{+0.70}$, and $\chi^{2} /$ d.o.f. $\simeq 45.6 / 49$. The reported $E W$ s of $\mathrm{Fe} \mathrm{K} \alpha$ for these two models are $1.49_{-1.27}^{+11.11} \mathrm{keV}$ and $3.13_{-2.00}^{+2.27} \mathrm{keV}$, respectively. We present the first XMM-Newton X-ray spectrum of NGC 2639 and find that the best fit consists of an absorbed powerlaw $\left(N_{\mathrm{H}} \simeq 1.62_{-1.56}^{+1.76} \times 10^{21} \mathrm{~cm}^{-2}, \Gamma \simeq 2.21_{-1.02}^{+1.83}\right)$ and a soft component modeled with a thermal plasma (MEKAL in XSPEC) of temperature $k T \simeq 0.63_{-0.09}^{+0.12}$. We do not detect the Fe $\mathrm{K} \alpha$ line in the XMM-Newton pn spectrum probably due to the lack of enough counts above $2.0 \mathrm{keV}$.

\section{NGC 4151}

NGC 4151 is one of the most extensively studied Seyfert galaxies in nearly all wavelengths. The ASCA and BeppoSAX X-ray spectra were fitted with a flat absorbed powerlaw $(\Gamma \simeq 1.65)$, contribution from both a cold reflector and a two-component absorber, an intrinsic neutral component with $N_{\mathrm{H}} \simeq 3.4 \times$ $10^{22} \mathrm{~cm}^{-2}$, and a highly ionized absorber with $N_{\mathrm{H}} \simeq 2.4 \times$ $10^{23} \mathrm{~cm}^{-2}$ (Schurch \& Warwick 2002; Piro et al. 2005). Yang et al. (2001) report the Chandra ACIS observation of NGC 4151 with notably extended soft X-ray emission on a scale of several hundred parsecs and a spatially unresolved hard X-ray $(\geq 2.0 \mathrm{keV})$ component. The spectrum of the unresolved nuclear source is described by a heavily absorbed $\left(N_{\mathrm{H}} \sim 10^{22} \mathrm{~cm}^{-2}\right)$, hard powerlaw $(\Gamma \simeq 0.3)$, and soft emission from either a powerlaw $(\Gamma \simeq 2.6)$ or a thermal $(k T \simeq 0.6 \mathrm{keV})$ component. We fitted the XMM-Newton pn spectrum with a model consisting of: a soft component characterized by a powerlaw and a thermal plasma model with a temperature of $k T \simeq 0.13 \mathrm{keV}$; and an emission line at $0.88 \mathrm{keV}$; a hard component characterized with an absorbed powerlaw $\left(N_{\mathrm{H}} \simeq 1.8 \times 10^{21} \mathrm{~cm}^{-2}, \Gamma \simeq 1.75_{-0.03}^{+0.06}\right)$, accompanied by warm absorption $\left(N_{\mathrm{H}} \simeq 5.2_{-1.20}^{+1.45} \times 10^{22} \mathrm{~cm}^{-2}\right)$ and partial covering $\left(N_{\mathrm{H}} \simeq 9.06_{-0.98}^{+1.27} \times 10^{22} \mathrm{~cm}^{-2}\right.$, covering fraction $f \simeq 0.69_{-0.05}^{+0.04}$; and a reflection component; and an Fe K $\alpha$ emission line at $6.4 \mathrm{keV}$. The $\mathrm{Fe} \mathrm{K} \alpha$ line is fitted with a narrow Gaussian component with an $E W \simeq 108.4_{-6.7}^{+6.5} \mathrm{eV}$. 


\section{MRK 766}

RoSAT and ASCA observations have shown that the X-ray spectrum of MRK 766 can be described by a powerlaw of index 1.6-2.0 (increasing strongly with flux), a reflection component, and a narrow $\mathrm{Fe} \mathrm{K} \alpha$ emission line $(E W \sim 100 \mathrm{eV})$ (Leighly et al. 1996). Later observations with BeppoSAX found a steeper powerlaw $(\Gamma \simeq 2.2)$ and evidence of an absorption edge at $7.4 \mathrm{keV}$ (Matt et al. 2000b). Using XMM-Newton observations Miller et al. (2006) report the variations in the flux of $\mathrm{Fe} \mathrm{K} \alpha$ line on short time scales $(5-20 \mathrm{ks})$ and its strong correlation with the continuum emission. We fitted the XMM-Newton pn X-ray spectrum of MRK 766 with a less absorbed fairly steep powerlaw $\left(\Gamma \simeq 2.17_{-0.08}^{+0.10}, N_{\mathrm{H}}\right.$ fixed to Galactic value) with partial covering $\left(N_{\mathrm{H}} \simeq 6.42_{-0.56}^{+0.93} \times 10^{22} \mathrm{~cm}^{-2}\right.$, covering fraction $\left.f \simeq 0.51_{-0.03}^{+0.02}\right)$ and warm absorption $\left(N_{\mathrm{H}} \simeq 2.1_{-0.24}^{+0.28} \times 10^{21} \mathrm{~cm}^{-2}\right)$, a reflection component at the hard end, a soft component fitted with a thermal plasma model of temperature $k T \sim 0.2 \mathrm{keV}$, and narrow Gaussian emission lines at $0.48 \mathrm{keV}, 6.44 \mathrm{keV}$, and $6.67 \mathrm{keV}$. The $\mathrm{Fe} \mathrm{K} \alpha$ emission may have broad component, but we get a better fit with a narrow emission line with centroid energy at $6.44 \mathrm{keV}$ and a second narrow line at $6.67 \mathrm{keV}$. The second line could be $\mathrm{Fe} \mathrm{K} \alpha$ from highly ionized (Fe XXV) material or a $\mathrm{K}_{\beta}$ line from neutral matter.

\section{MRK 231}

X-ray observations by ROSAT, ASCA (Turner 1999; Iwasawa 1999; Maloney \& Reynolds 2000), and more recently Chandra (Gallagher et al. 2002; Ptak et al. 2003) have revealed the presence of extended soft X-ray emission with a thermal origin that is likely to be associated with the circumnuclear starburst and a hard and flat $(\Gamma \simeq 0.7)$ powerlaw component, as well as an Fe $\mathrm{K}_{\alpha}$ emission line with $E W \sim 300 \mathrm{eV}$ (the line was detected by ASCA but not by Chandra). From combined XMMNewton and BeppoSAX observations of MRK 231, Braito et al. (2004) find a highly absorbed $\left(N_{\mathrm{H}} \sim 2 \times 10^{24} \mathrm{~cm}^{-2}\right)$ AGN component. We obtain the best fit with an absorbed powerlaw $\left(N_{\mathrm{H}} \simeq 4.6_{-1.6}^{+1.4} \times 10^{21} \mathrm{~cm}^{-2}, \Gamma \simeq 1.57_{-0.30}^{+0.14}\right)$ accompanied by a partial covering $\left(N_{\mathrm{H}} \simeq 8.35_{-4.64}^{+4.02} \times 10^{22} \mathrm{~cm}^{-2}\right.$, covering fraction $f \simeq 0.74_{-0.25}^{+0.09}$ ), a soft component fitted with two thermal plasma models with temperatures $k T \simeq 0.33 \mathrm{keV}$ and $0.99 \mathrm{keV}$, and narrow emission lines at $6.28 \mathrm{keV}$ and $6.66 \mathrm{keV}$. The soft X-ray component may make a significant contribution from starburst activity (Braito et al. 2004).

\section{ARK 564}

Vignali et al. (2004) analyzed two sets of XMM-Newton observations of ARK 564 taken a year apart (2000 June and 2001 June) and fitted the $0.6-10.0 \mathrm{keV}$ continuum by a soft blackbody component $(k T \simeq 140-150 \mathrm{eV})$, a steep powerlaw $(\Gamma \simeq 2.50-2.55)$, and an absorption edge at a rest-frame energy of $\sim 0.73 \mathrm{keV}$, corresponding to O VII. No significant spectral changes were observed between the two observations, although the X-ray flux in the second observation is $40 \%-50 \%$ lower. We obtain the best fit to the $0.6-10.0 \mathrm{keV} X M M-N e w t o n$ pn spectrum by a less absorbed steep powerlaw ( $\Gamma \simeq 2.32_{-0.10}^{+0.08}, N_{\mathrm{H}}$ fixed to the Galactic value) with partial covering $\left(N_{\mathrm{H}} \simeq 3.09_{-0.45}^{+0.52} \times 10^{22} \mathrm{~cm}^{-2}\right.$, covering fraction $\left.f \simeq 0.61_{-0.15}^{+0.26}\right)$, a soft component fitted with a steep powerlaw $\left(\Gamma \simeq 3.42_{-0.12}^{+0.13}\right)$, a thermal plasma model with temperature $k T \simeq 0.97 \mathrm{keV}$, and narrow emission lines at $6.33 \mathrm{keV}$ and $6.71 \mathrm{keV}$. While fitting, we excluded the $0.5-0.6 \mathrm{keV}$ part of the spectrum since these data are of poor quality, making it difficult to obtain even a reasonably good fit.

\section{NGC 7469}

Blustin et al. (2003) fitted the 0.2-10.0 keV XMM-Newton EPIC pn spectrum with a combination of a Galactic absorbed powerlaw $(\Gamma \simeq 1.75)$, two blackbody models, and a narrow $\mathrm{Fe} \mathrm{K} \alpha$ emission line. They noted significant residuals at low energies for which they suggest an instrumental origin. We fitted the 0.5-10.0 XMM-Newton pn spectrum with a less absorbed powerlaw ( $\Gamma \simeq 2.04_{-0.02}^{+0.02}, N_{\mathrm{H}}$ fixed to Galactic value), a partial covering $\left(N_{\mathrm{H}} \simeq 35.55_{-6.77}^{+9.93} \times 10^{22} \mathrm{~cm}^{-2}\right.$, the covering fraction $f \simeq 0.28_{-0.03}^{+0.03}$, a soft component fitted with a thermal plasma model of temperature $k T \simeq 0.18 \mathrm{keV}$, and a narrow Gaussian fitted to the $\mathrm{Fe} \mathrm{K} \alpha$ line. Using ASCA observations, Reynolds (1997) reports both narrow and broad components to the Fe line, which is supported by De Rosa et al. (2002) using BeppoSAX data, and Nandra et al. (1997) has suggested that it was relativistically broadened. We fit the $\mathrm{Fe} \mathrm{K} \alpha$ line with a narrow Gaussian component, however, and the data do not need a broad component for a good fit, consistent with Blustin et al. (2003).

\section{MRK 926}

Bianchi et al. (2004) fitted the combined 2.5-220 keV XMM-Newton and BeppoSAX spectrum with a baseline model consisting of a powerlaw $\left(\Gamma \simeq 1.72_{-0.06}^{+0.08}\right)$ with intrinsic absorption over and above Galactic, a reflection component, and a Gaussian to reproduce the Fe $\mathrm{K} \alpha$ line $\left(E W \simeq 45_{-24}^{+85} \mathrm{eV}\right)$. They also report an upper limit to the $\mathrm{Fe} \mathrm{K}_{\beta}$ flux of the order of the $\mathrm{K} \alpha$ flux, and suggest the possibility of a significant contribution from an Fe XXVI line. Weaver et al. (2001) report strong line variability, both in centroid and in flux, between three ASCA observations. We fitted the $0.5-10.0 \mathrm{keV} \mathrm{XMM-Newton} \mathrm{spectrum}$ with an absorbed partially covered powerlaw $\left(\Gamma \simeq 1.98_{-0.02}^{+0.03}, N_{\mathrm{H}}\right.$ fixed to Galactic value, $N_{\text {part, } \mathrm{H}} \simeq 4.35_{-1.99}^{+2.86} \times 10^{22} \mathrm{~cm}^{-2}$, covering fraction $f \simeq 0.15_{-0.06}^{+0.06}$, a reflection component, and a narrow Gaussian to fit the $\mathrm{Fe} \mathrm{K} \alpha$ line. Notably, we do not see any soft excess in this Seyfert.

\section{MRK 530}

MRK 530 has not been observed by either Chandra or ASCA. We present the XMM-Newton pn spectrum of MRK 530 for the first time. We fitted the $0.5-10.0 \mathrm{keV}$ spectrum by an absorbed partially covered powerlaw $\left(\Gamma \simeq 2.28_{-0.01}^{+0.03}, N_{\mathrm{H}}\right.$ fixed to the Galactic value, $N_{\text {part, } \mathrm{H}} \simeq 14.81_{-4.18}^{+5.62} \times 10^{22} \mathrm{~cm}^{-2}$, covering fraction $f \simeq 0.27_{-0.27}^{+0.27}$ ), a soft component modeled with thermal plasma at a temperature $k T \simeq 0.20 \mathrm{keV}$, and a narrow Gaussian fitted to the $\mathrm{Fe} \mathrm{K} \alpha$ line.

\section{MRK 348}

Awaki et al. (2006) fitted the XMM-Newton pn $0.2-10.0 \mathrm{keV}$ spectrum of MRK 348 with an absorbed powerlaw $\left(N_{\mathrm{H}} \simeq\right.$ $\left.13.5 \pm 0.02 \times 10^{22} \mathrm{~cm}^{-2}, \Gamma \simeq 1.61 \pm 0.02\right)$, a soft component fitted with a thermal plasma model with temperature $k T \simeq 0.56_{-0.02}^{+0.01} \mathrm{keV}$, and an Fe K $\alpha$ line $(E W \sim 46.4 \pm 20 \mathrm{eV})$, and obtain $\chi^{2} /$ d.o.f. $\sim 1.46$. They fixed the metal abundance of the thermal plasma at 0.1 times the solar abundance, the characteristic value of a normal galaxy (Terashima et al. 2002). 
We used the same XMM-Newton data and find the best fit to the $0.5-10.0 \mathrm{keV}$ spectrum consists of an absorbed powerlaw $\left(N_{\mathrm{H}} \simeq 6.86_{-1.01}^{+0.84} \times 10^{22} \mathrm{~cm}^{-2}, \Gamma \simeq 1.70_{-0.06}^{+0.07}\right)$ with partial covering $\left(N_{\mathrm{H}, \text { part }} \simeq 10.47_{-1.00}^{+0.68} \times 10^{22} \mathrm{~cm}^{-2}\right.$, covering fraction $\left.f \simeq 0.84_{-0.06}^{+0.06}\right)$, a soft component fitted with a powerlaw $\left(\Gamma \simeq 2.75_{-0.16}^{+0.17}\right)$, and a narrow Gaussian fitted to $\mathrm{Fe} \mathrm{K} \alpha$ line $\left(E W \sim 34.2_{-10.4}^{+10.8}\right)$ to obtain $\chi^{2} /$ d.o.f. $\sim 1.05$.

\section{MRK 1}

Guainazzi et al. (2005b) fitted the 0.5-10.0 keV XMM-Newton pn spectrum of MRK 1 with a highly absorbed powerlaw $\left(N_{\mathrm{H}} \geq\right.$ $\left.110 \times 10^{22} \mathrm{~cm}^{-2}, \Gamma \simeq 2.41_{-0.11}^{+0.13}\right)$ and a scattered component with partial covering factor fixed to 1 , and obtained $\chi^{2} /$ d.o.f $\sim 36 / 32$. They report several emission lines including iron $\mathrm{K}$ shell lines in $0.5-2.0 \mathrm{keV}$ band but no thermal component. To constrain the $\mathrm{Fe} \mathrm{K} \alpha$ line, they performed both global and local fits with centroid energy fixed at $6.4 \mathrm{keV}$, and find upper limits to the equivalent width of $\sim 800 \mathrm{eV}$ and $\sim 2.0 \mathrm{keV}$, respectively. Due to the relatively low counts in the data, we used C-statistics to fit the spectrum and find the best fit consists of a hard component $(2.0-10.0 \mathrm{keV})$ that is completely dominated by reflection $(\Gamma \simeq$ 2.0), a soft component fitted with a powerlaw $\left(\Gamma \simeq 2.55_{-0.29}^{+0.30}\right)$, a thermal plasma model $\left(k T \simeq 0.82_{-0.09}^{+0.08} \mathrm{keV}\right)$, and a Gaussian fitted to the Fe $\mathrm{K} \alpha$ line $\left(E W \sim 1.25_{-0.86}^{+1.29} \mathrm{keV}\right)$. The reflectiondominated spectrum, high $E W$ of the Fe line, and the low ratio of hard X-ray to [OIII] flux suggest that MRK 1 is likely to be a Compton-thick source.

\section{NGC 2273}

Guainazzi et al. (2005a) attempted to fit the XMM-Newton spectrum of NGC 2273 with a family of models in which a hard X-ray component is accounted for by an absorbed powerlaw but yields an unacceptable flat intrinsic spectral index $(\Gamma \simeq-0.2--0.5)$ and also a large $E W(\simeq 2.0-3.6 \mathrm{keV})$ of the $\mathrm{Fe} \mathrm{K} \alpha$ line with respect to the measured column density $\left(N_{\mathrm{H}} \simeq 1.4-12.0 \times 10^{22} \mathrm{~cm}^{-2}\right)$, and suggest that the spectrum is dominated by Compton reflection. In the best fit reported by Guainazzi et al. (2005a), the soft X-ray spectrum is accounted for by thermal emission model with temperature $k T \simeq 0.8 \pm 0.2 \mathrm{keV}$, hard $\mathrm{X}$-ray fitted by reflection model $\left(\Gamma_{\text {hard }} \simeq 1.5 \pm 0.4, N_{\mathrm{H}} \geq 18.0 \times 10^{23} \mathrm{~cm}^{-2}\right)$, and an Fe K $\alpha$ line with $E W \simeq 2.2_{-0.3}^{+0.4} \mathrm{keV}$. We find the best fit in which the hard component is completely accounted for by reflection component ( $\Gamma \simeq 0.67_{-0.92}^{+0.37}$, PEXRAV model), and the soft component is fitted with a powerlaw $\left(\Gamma \simeq 2.79_{-0.82}^{+1.87}\right)$ and a Gaussian line fitted to Fe $\mathrm{K} \alpha\left(E W \sim 2.18_{-0.44}^{+0.45} \mathrm{keV}\right)$. The Compton-thick nature of this source is evident from the reflection-dominated spectrum, high $E W$ of $\mathrm{Fe} \mathrm{K} \alpha$ line, and low flux ratio of hard X-ray to [OIII].

\section{MRK 78}

MRK 78 has not been observed by Chandra or ASCA. Levenson et al. (2001) fitted its RoSAT PSPC soft X-ray spectrum with a thermal plasma $(k T \simeq 0.76 \mathrm{keV})$ model absorbed by the Galactic column density and report extended soft X-ray emission indicative of a circumnuclear starburst. We have presented the XMMNewton spectrum of MRK 78 for the first time. Our best fit has the hard component completely accounted for by a reflection component $\left(\Gamma \simeq 1.01_{-0.54}^{+0.44}\right.$, "pexrav" model), the soft component fitted with a very steep powerlaw $\left(\Gamma \simeq 7.05_{-1.84}^{+1.89}\right)$, and a Gaussian fitted to $\mathrm{Fe} \mathrm{K} \alpha(E W \sim 0.67 \mathrm{keV})$. All the spectral components are absorbed by an equivalent hydrogen column density of $N_{\mathrm{H}} \simeq 6.54_{-2.5}^{+3.0} \times 10^{21} \mathrm{~cm}^{-2}$. The predominance of the reflection component, high $E W$ of the $\mathrm{Fe} \mathrm{K} \alpha$ line, and the ratio of hard $\mathrm{X}$-ray flux to [OIII] line flux ratio suggest that obscuration is nearly Compton-thick.

\section{NGC 5135}

NGC 5135 has not been observed by XMM-Newton, so we used the Chandra spectral properties given in Guainazzi et al. (2005a) for our study. Both Guainazzi et al. (2005a) and Levenson et al. (2004) obtained very similar spectral fits consisting of a thermal-plus-reflection model. The soft X-ray spectrum requires two thermal components with $k T \simeq 80 \mathrm{eV}$ and $k T \simeq$ $390 \mathrm{eV}$ and an additional emission line with centroid energy $E_{\mathrm{c}} \simeq 1.78 \mathrm{keV}$. Above $2.0 \mathrm{keV}$ the spectrum is dominated by Compton-reflection, consistent with the AGN being obscured by a column density $N_{\mathrm{H}} \geq 9.0 \times 10^{23} \mathrm{~cm}^{-2}$ (for an intrinsic photon index of 1.5 and a reflection fraction $\leq 0.5$ ). The $E W$ of $\mathrm{Fe} \mathrm{K} \alpha$ fluorescent emission line against the reflection continuum is $1.7_{-0.8}^{+0.6} \mathrm{keV}$. Using Chandra observations, Levenson et al. (2004) report a circumnuclear starburst in NGC 5135 and could spatially isolate the AGN emission that is entirely obscured by the column density of $N_{\mathrm{H}} \geq 10^{24} \mathrm{~cm}^{-2}$, detectable in the Chandra bandpass only as a strongly reprocessed weak continuum represented by a flat powerlaw $(\Gamma \simeq 0)$ plus a prominent iron $\mathrm{K}_{\alpha}$ emission line with an $E W$ of $\sim 2.4 \mathrm{keV}$. We confirm the Compton-thick obscuration in NGC 5135 using broad-band 0.5-50 keV Suzaku observations (Singh et al. 2011, in prep.).

\section{MRK 477}

MRK 477 has not been observed by XMM-Newton or Chandra, we used the ASCA spectral properties given in Levenson et al. (2001) for our study. The best fit reported by Levenson et al. (2001) consists of an absorbed powerlaw $\left(N_{\mathrm{H}} \simeq\right.$ $2.4_{-1.2}^{+1.7} \times 10^{23} \mathrm{~cm}^{-2}, \Gamma \simeq 1.9$ fixed) and an unresolved $\mathrm{Fe} \mathrm{K} \alpha$ emission line of $E W \sim 560_{-500}^{+560} \mathrm{eV}$. The addition of a thermal component $k T \sim 0.9 \mathrm{keV}$ to this model rendered the reasonable spectral parameter values but did not statistically improve the fit. Heckman et al. (1997) pointed out that MRK 477 has a powerful circumnuclear starburst with the bolometric luminosity of $\sim 3 \times 10^{10}-10^{11} L_{\odot}$. This luminosity is comparable to the AGN activity. However, Levenson et al. (2001) conclude that the soft $\mathrm{X}$-ray spectrum is dominated by the AGN.

\section{NGC 5929}

NGC 5929 has not been observed by XMM-Newton or Chandra. Cardamone et al. (2007) have modeled the 0.5-8.0 keV ASCA spectrum as a sum of three components: a weakly absorbed $\left(N_{\mathrm{H}, 1} \simeq 5.16 \times 10^{21} \mathrm{~cm}^{-2}\right)$ powerlaw with photon index $\Gamma \simeq 1.70$, a heavily absorbed powerlaw $\left(N_{\mathrm{H}, 2} \simeq 2.77 \times 10^{23} \mathrm{~cm}^{-2}\right)$ with the same photon index and a Gaussian Fe K $\alpha$ line of $E W$ of $0.35 \mathrm{keV}$ centered at $6.19 \mathrm{keV}$. We used the ASCA spectral properties of NGC 5929 for our study. 


\section{NGC 7212}

Guainazzi et al. (2005b) fitted the $0.5-10.0 \mathrm{keV} X M M-N e w t o n$ spectrum with a heavily absorbed powerlaw $\left(N_{\mathrm{H}} \geq 160 \times\right.$ $\left.10^{22} \mathrm{~cm}^{-2}, \Gamma \simeq 1.5_{-0.6}^{+0.3}\right)$, two thermal plasma components with $k T \simeq 0.16$ and $0.72 \mathrm{keV}$, and an Fe $\mathrm{K} \alpha$ line to obtain $\chi^{2} /$ d.o.f. $\sim 97.0 / 65$. The Fe K $\alpha$ line was fitted both globally and locally, giving $E W \mathrm{~s} \sim 900_{-300}^{+200} \mathrm{eV}$ and $\sim 1100 \pm 200 \mathrm{eV}$, respectively. We fitted the $0.5-10.0 \mathrm{keV} X M M-N e w t o n$ spectrum with the hard component completely accounted for by reflection $\left(\Gamma \simeq 0.99_{-0.37}^{+0.54}\right)$, the soft component fitted with a powerlaw $\left(\Gamma \simeq 2.32_{-0.20}^{+0.23}\right)$, a narrow Gaussian fitted to the emission line feature at $\sim 0.9 \mathrm{keV}$, and another Gaussian fitted to the Fe K $\alpha$ emission line at $6.4 \mathrm{keV}$ giving $E W \sim 0.71_{-0.13}^{+0.25} \mathrm{keV}$.

\section{MRK 533}

MRK 533 has not been observed by Chandra or ASCA. Levenson et al. (2001) fitted its ROSAT/PSPC soft X-ray spectrum with a powerlaw $\left(\Gamma \simeq 2.0_{-1.3}^{+0.7}\right)$. We presented the $X M M$ Newton pn spectrum of MRK 533 for the first time. We fitted the 0.5-10.0 keV spectrum with a model in which the soft component is fitted with a powerlaw $\left(\Gamma \simeq 3.75_{-0.61}^{+1.45}\right)$ and thermal plasma emission with the temperature $k T \simeq 0.76_{-0.10}^{+0.13}$; a hard component completely accounted for by reflection $\left(\Gamma \simeq 2.12_{-0.51}^{+0.49}\right.$, PEXRAV model); and narrow Gaussians fitted to the $\mathrm{Fe} \mathrm{K} \alpha$ line at $6.4 \mathrm{keV}$; and the $\mathrm{Fe} \mathrm{K}_{\beta}$ line at $7.04 \mathrm{keV}$, with $E W \mathrm{~s} \sim 0.56_{-0.29}^{+0.24} \mathrm{keV}$ and $\sim 0.67_{-0.33}^{+0.39} \mathrm{keV}$, respectively. Our X-ray spectral properties suggest for the Compton-thick obscuration in MRK 533.

\section{NGC 7682}

We have presented the XMM-Newton spectrum of NGC 7682 for the first time and find the best fit consists of a soft component fitted with a steep powerlaw $\left(\Gamma \simeq 5.36_{-2.38}^{+2.78}\right)$; a hard component completely accounted for by reflection $\left(\Gamma \simeq 1.77_{-0.61}^{+0.58}\right.$, PEXRAV model); and narrow Gaussians fitted to the $\mathrm{Fe} \mathrm{K} \alpha$ line at $6.4 \mathrm{keV}$ and $\mathrm{Fe} \mathrm{K}_{\beta}$ line at $7.11 \mathrm{keV}$ of $E W \mathrm{~s} \sim 0.48_{-0.31}^{+0.31} \mathrm{keV}$ and $\sim 0.46_{-0.40}^{+0.46} \mathrm{keV}$, respectively. All the spectral components are absorbed by an equivalent hydrogen column density $N_{\mathrm{H}} \simeq$ $3.48_{-2.51}^{+5.78} \times 10^{21} \mathrm{~cm}^{-2}$. Our XMM-Newton X-ray spectral properties suggest for the Compton-thick obscuration in NGC 7682.

\section{References}

Akylas, A., \& Georgantopoulos, I. 2009, A\&A, 500, 999 Antonucci, R. 1993, ARA\&A, 31, 473

Antonucci, R. 2002, in AGN Surveys, ed. R. F. Green, E. Y. Khachikian, \& D. B. Sanders, IAU Coll., 184, ASP Conf. Ser., 284, 147 Antonucci, R. R. J., \& Miller, J. S. 1985, ApJ, 297, 621

Arnaud, K. A. 1996, in Astronomical Data Analysis Software and Systems V, ed. G. H. Jacoby, \& J. Barnes, ASP Conf. Ser., 10117

Awaki, H., Koyama, K., Inoue, H., \& Halpern, J. P. 1991, PASJ, 43, 195 Awaki, H., Ueno, S., Taniguchi, Y., \& Weaver, K. A. 2000, ApJ, 542, 175 Awaki, H., Murakami, H., Ogawa, Y., \& Leighly, K. M. 2006, ApJ, 645, 928 Awaki, H., Terashima, Y., Higaki, Y., \& Fukazawa, Y. 2009, PASJ, 61, 317 Bassani, L., Dadina, M., Maiolino, R., et al. 1999, ApJS, 121, 473 Beckmann, V., Soldi, S., Ricci, C., et al. 2009, A\&A, 505, 417

Bianchi, S., Matt, G., Balestra, I., Guainazzi, M., \& Perola, G. C. 2004, A\&A, 422, 65

Bianchi, S., Guainazzi, M., Matt, G., et al. 2005a, A\&A, 442, 185

Bianchi, S., Miniutti, G., Fabian, A. C., \& Iwasawa, K. 2005b, MNRAS, 360, 380

Blustin, A. J., Branduardi-Raymont, G., Behar, E., et al. 2003, A\&A, 403, 481 Blustin, A. J., Kriss, G. A., Holczer, T., et al. 2007, A\&A, 466, 107
Braito, V., Della Ceca, R., Piconcelli, E., et al. 2004, A\&A, 420, 79

Buchanan, C. L., Gallimore, J. F., O'Dea, C. P., et al. 2006, AJ, 132, 401

Cappi, M., Panessa, F., Bassani, L., et al. 2006, A\&A, 446, 459

Cardamone, C. N., Moran, E. C., \& Kay, L. E. 2007, AJ, 134, 1263

Cid Fernandes, R. J., Storchi-Bergmann, T., \& Schmitt, H. R. 1998, MNRAS, 297, 579

Dadina, M. 2008, A\&A, 485, 417

Dahari, O., \& De Robertis, M. M. 1988, ApJ, 331, 727

De Rosa, A., Fabian, A. C., \& Piro, L. 2002, MNRAS, 334, L21

Ferruit, P., Wilson, A. S., \& Mulchaey, J. 2000, ApJS, 128, 139

Gallagher, S. C., Brandt, W. N., Chartas, G., Garmire, G. P., \& Sambruna, R. M. 2002, ApJ, 569, 655

Ghisellini, G., George, I. M., Fabian, A. C., \& Done, C. 1991, MNRAS, 248, 14 Ghisellini, G., Haardt, F., \& Matt, G. 1994, MNRAS, 267, 743

Gilli, R., Comastri, A., \& Hasinger, G. 2007, A\&A, 463, 79

González Delgado, R. M., Heckman, T., \& Leitherer, C. 2001, ApJ, 546, 845

Grandi, P., Haardt, F., Ghisellini, G., et al. 1998, ApJ, 498, 220

Griffiths, R. G., Warwick, R. S., Georgantopoulos, I., Done, C., \& Smith, D. A. 1998, MNRAS, 298, 1159

Guainazzi, M., Fabian, A. C., Iwasawa, K., Matt, G., \& Fiore, F. 2005a, MNRAS, 356,295

Guainazzi, M., Matt, G., \& Perola, G. C. 2005b, A\&A, 444, 119

Heckman, T. M., Gonzalez-Delgado, R., Leitherer, C., et al. 1997, ApJ, 482, 114

Heckman, T. M., Ptak, A., Hornschemeier, A., \& Kauffmann, G. 2005, ApJ, 634, 161

Ho, L. C., \& Ulvestad, J. S. 2001, ApJS, 133, 77

Kellermann, K. I., Sramek, R., Schmidt, M., Shaffer, D. B., \& Green, R. 1989, AJ, 98, 1195

King, A. 2005, ApJ, 635, L121

Kinkhabwala, A., Sako, M., Behar, E., et al. 2002, ApJ, 575, 732

Krolik, J. H., Madau, P., \& Zycki, P. T. 1994, ApJ, 420, L57

Lal, D. V., Shastri, P., \& Gabuzda, D. C. 2011, ApJ, 731, 68

Leahy, D. A., \& Creighton, J. 1993, MNRAS, 263, 314

Leighly, K. M., Mushotzky, R. F., Yaqoob, T., Kunieda, H., \& Edelson, R. 1996, ApJ, 469, 147

Levenson, N. A., Weaver, K. A., \& Heckman, T. M. 2001, ApJ, 550, 230

Levenson, N. A., Weaver, K. A., Heckman, T. M., Awaki, H., \& Terashima, Y. 2004, ApJ, 602, 135

Levenson, N. A., Heckman, T. M., Krolik, J. H., Weaver, K. A., \& Życki, P. T. 2006, ApJ, 648, 111

Magdziarz, P., \& Zdziarski, A. A. 1995, MNRAS, 273, 837

Maiolino, R., Ruiz, M., Rieke, G. H., \& Papadopoulos, P. 1997, ApJ, 485, 552

Maiolino, R., Salvati, M., Bassani, L., et al. 1998, A\&A, 338, 781

Malkan, M. A., Gorjian, V., \& Tam, R. 1998, ApJS, 117, 25

Mason, K. O., Branduardi-Raymont, G., Ogle, P. M., et al. 2003, ApJ, 582, 95

Mateos, S., Carrera, F. J., Page, M. J., et al. 2010, A\&A, 510, A35

Matt, G., Fiore, F., Perola, G. C., et al. 1996, MNRAS, 281, L69

Matt, G., Fabian, A. C., Guainazzi, M., et al. 2000a, MNRAS, 318, 173

Matt, G., Perola, G. C., Fiore, F., et al. 2000b, A\&A, 363, 863

Matt, G., Bianchi, S., de Rosa, A., Grandi, P., \& Perola, G. C. 2006, A\&A, 445, 451

Miller, L., Turner, T. J., Reeves, J. N., et al. 2006, A\&A, 453, L13

Moran, E. C., Filippenko, A. V., \& Kay, L. E. 2000, BAAS, 32, 1182

Mulchaey, J. S., Koratkar, A., Ward, M. J., et al. 1994, ApJ, 436, 586

Mulchaey, J. S., Wilson, A. S., \& Tsvetanov, Z. 1996, ApJ, 467, 197

Nandra, K., \& Pounds, K. A. 1994, MNRAS, 268, 405

Nandra, K., George, I. M., Mushotzky, R. F., Turner, T. J., \& Yaqoob, T. 1997, ApJ, 477, 602

Nelson, C. H., \& Whittle, M. 1995, ApJS, 99, 67

Osterbrock, D. E., \& Ferland, G. J. 2006, Astrophysics of gaseous nebulae and active galactic nuclei, ed. D. E. Osterbrock, \& G. J. Ferland

Panessa, F., \& Bassani, L. 2002, A\&A, 394, 435

Panessa, F., Bassani, L., Cappi, M., et al. 2006, A\&A, 455, 173

Perola, G. C., Matt, G., Fiore, F., et al. 2000, A\&A, 358, 117

Piro, L., de Rosa, A., Matt, G., \& Perola, G. C. 2005, A\&A, 441, L13

Polletta, M., Bassani, L., Malaguti, G., Palumbo, G. G. C., \& Caroli, E. 1996, ApJS, 106, 399

Pounds, K. A., \& Page, K. L. 2005, MNRAS, 360, 1123

Ptak, A., Heckman, T., Levenson, N. A., Weaver, K., \& Strickland, D. 2003, ApJ, 592, 782

Reynolds, C. S. 1997, MNRAS, 286, 513

Risaliti, G., Maiolino, R., \& Salvati, M. 1999, ApJ, 522, 157

Sako, M., Kahn, S. M., Paerels, F., \& Liedahl, D. A. 2000, ApJ, 543, L115

Sambruna, R. M., Netzer, H., Kaspi, S., et al. 2001, ApJ, 546, L13

Schmidt, M., \& Green, R. F. 1983, ApJ, 269, 352

Schmitt, H. R., Donley, J. L., Antonucci, R. R. J., Hutchings, J. B., \& Kinney, A. L. 2003, ApJS, 148, 327

Schurch, N. J., \& Warwick, R. S. 2002, MNRAS, 334, 811

A84, page 14 of 15 
V. Singh et al.: X-ray spectral properties of Seyfert galaxies and the unification scheme

Schurch, N. J., Warwick, R. S., Griffiths, R. E., \& Kahn, S. M. 2004, MNRAS, 350,1

Smith, D. A., \& Done, C. 1996, MNRAS, 280, 355

Terashima, Y., Iyomoto, N., Ho, L. C., \& Ptak, A. F. 2002, ApJS, 139, 1

Tran, H. D. 2001, ApJ, 554, L19

Tran, H. D. 2003, ApJ, 583, 632

Treister, E., Urry, C. M., \& Virani, S. 2009, ApJ, 696, 110

Tueller, J., Mushotzky, R. F., Barthelmy, S., et al. 2008, ApJ, 681, 113

Turner, T. J., George, I. M., Nandra, K., \& Mushotzky, R. F. 1997a, ApJS, 113, 23

Turner, T. J., George, I. M., Nandra, K., \& Mushotzky, R. F. 1997b, ApJ, 488, 164

Turner, T. J., George, I. M., Nandra, K., \& Mushotzky, R. F. 1998, ApJ, 493, 91
Urry, K., Padovanni, G., Wilson, A. S., \& Yoshida, M. 1995, ApJ, 521, 565 Vignali, C., Brandt, W. N., Boller, T., Fabian, A. C., \& Vaughan, S. 2004, MNRAS, 347, 854

Wang, J., Mao, Y. F., \& Wei, J. Y. 2009, AJ, 137, 3388

Weaver, K. A., Nousek, J., Yaqoob, T., et al. 1996, ApJ, 458, 160

Weaver, K. A., Gelbord, J., \& Yaqoob, T. 2001, ApJ, 550, 261

Weedman, D. W. 1977, ARA\&A, 15, 69

Whittle, M. 1992, ApJS, 79, 49

Worsley, M. A., Fabian, A. C., Bauer, F. E., et al. 2005, MNRAS, 357, 1281

Yang, Y., Wilson, A. S., \& Ferruit, P. 2001, ApJ, 563, 124

Yee, H. K. C., Stockman, H. S., \& Green, R. F. 1981, BAAS, 13, 806

Young, A. J., Wilson, A. S., \& Shopbell, P. L. 2001, ApJ, 556, 6 